\title{
Aging and strategic prospective memory monitoring
}

\author{
B. Hunter Ball ${ }^{1,2} \cdot$ Y. Peeta $\mathrm{Li}^{2} \cdot$ Julie M. Bugg ${ }^{2}$
}

Published online: 18 October 2019

(C) The Psychonomic Society, Inc. 2019

\begin{abstract}
Monitoring the environment for the occurrence of prospective memory (PM) targets is a resource-demanding process that produces cost (e.g., slowing) to ongoing activities. Prior research has shown that older adults are able to monitor strategically, which involves the activation of monitoring when contextually appropriate and deactivation of monitoring when it is not thereby affording conservation of limited-capacity attentional resources. However, the time course and efficiency with which these processes operate with increased age are unknown. In the current study, participants performed an ongoing lexical decision task in which words/nonwords were blocked by font color in sets of ten trials (ten red trials followed by ten blue trials). Importantly, participants were informed that PM targets ("TOR" syllable) would only occur in red trials. Replicating previous work, both younger and older adults were successfully able to disengage monitoring upon encountering the unexpected (i.e., blue) context. However, while younger adults completely disengaged monitoring in the unexpected context, older adults continued to show monitoring across the majority of trials. Additionally, younger, but not older, adults showed a re-engagement of monitoring at the end of the unexpected context in preparation for the upcoming expected context. These findings suggest that while strategic monitoring generally remains intact with increased age, the disengagement and preparatory re-engagement of strategic monitoring may operate less optimally for older adults.
\end{abstract}

Keywords Aging $\cdot$ Prospective memory $\cdot$ Attention $\cdot$ Strategic monitoring $\cdot$ Context

\section{Introduction}

Event-based prospective memory (PM) refers to the ability to remember to execute future intentions (e.g., attend a doctor's appointment) in response to external cues (e.g., a doctor's office), often while busily engaged in ongoing activities (e.g., driving). Considerable research has demonstrated that monitoring the environment for the occurrence of these cues (i.e., targets) can interfere

Electronic supplementary material The online version of this article (https://doi.org/10.3758/s13421-019-00976-8) contains supplementary material, which is available to authorized users.

B. Hunter Ball

Hunter.Ball@uta.edu

1 Department of Psychology, University of Texas at Arlington, 501 Nedderman Drive, Arlington, TX 76109, USA

2 Department of Psychological and Brain Sciences, Washington University in St. Louis, CB1125, One Brookings Dr, St. Louis, MO 63130, USA with ongoing activities (Guynn, 2003; Marsh, Hicks, Cook, Hansen, \& Pallos, 2003; Smith, 2003). For example, actively searching for the doctor's office may cause one to drive more slowly. Consequently, it would be optimal to strategically deploy these monitoring resources. By using contextual information such as location, one can disengage monitoring (and thus drive faster) when far from the medical complex (e.g., near home) and then engage monitoring (and thus drive slower) upon approaching the medical complex. Although the small amount of research on this topic suggests that strategic monitoring ability remains intact with increased age (Ball \& Bugg, 2018a; Kominsky \& Reese-Melancon, 2017), the efficiency with which these processes operate in older adults is unknown. The current study seeks to answer this question by examining possible differences between younger and older adults in the time course of strategic-monitoring processes.

In a typical laboratory-based PM study, participants perform an ongoing task (e.g., lexical decision task) with PM targets embedded (e.g., "TOR" syllable). Prior to beginning the ongoing task, participants form an intention to perform a specific action (e.g., press "7") upon encountering the PM 
targets (e.g., press "7" when you see "TOR" within a word or nonword). Monitoring is inferred by showing that possessing an intention produces a cost (typically in terms of slowing) to ongoing task performance in the PM block compared to when the same task is performed in a control block without an intention (Smith, Hunt, \& McVay, 2007). This cost is generally thought to occur because the ongoing task and PM task draw on the same limited-capacity attentional resources, so as more resources are devoted to noticing PM targets, fewer are available for making ongoing task decisions (Ball \& Brewer, 2018; Marsh et al., 2003; but see Heathcote, Loft, \& Remington, 2015; Strickland, Loft, Remington, \& Heathcote, 2018). Importantly, older adults typically show worse target detection than younger adults in tasks that require monitoring (Kliegel, Jäger, \& Phillips, 2008; Uttl, 2008, 2011). This is thought to occur because age-related declines in executive attention (Braver \& West, 2008; Hasher \& Zacks, 1988; West, 1996) result in older adults having greater difficulty in devoting the necessary resources towards noticing PM targets while busily engaged in ongoing task processing (Rendell, McDaniel, Forbes, \& Einstein, 2007). The current study examined whether declines in executive attention also result in older adults having greater difficulty in reducing monitoring in contexts in which targets are not expected (i.e., monitoring strategically).

Strategic monitoring is typically examined by specifying that PM targets will only occur in one contextual dimension of ongoing task stimuli (e.g., color, word type, shape, location). For example, participants may additionally be instructed that the syllable "TOR" will occur in red trials (expected context) but not blue trials (unexpected context). This is analogous to expecting that a pharmacy will be encountered in a business plaza but not a residential area. The typical finding is that cost (slowing) due to possessing an intention is substantially reduced in unexpected (blue) contexts relative to expected (red) contexts (Ball, Brewer, Loft, \& Bowden, 2015; Ball \& Bugg, 2018a; Bugg \& Ball, 2017; Cohen, Jaudas, Hirschhorn, Sobin, \& Gollwitzer, 2012; Knight et al., 2011; Kuhlmann \& Rummel, 2014; Lourenço \& Maylor, 2014; Lourenço, White, \& Maylor, 2013; Marsh, Hicks, \& Cook, 2006; see Smith, 2016 for a review). These findings indicate that individuals are able to use contextual information to strategically increase and decrease monitoring to conserve processing resources when possible.

Central to the current study, Lourenço and Maylor (2014) examined strategic monitoring in a group of younger adults during an ongoing task that was blocked in sets of eight trials. The blocks alternated in a predictive fashion from one color context to another (e.g., eight red trials followed by eight blue trials, and so forth). Participants were instructed that the PM target would occur in red trials (expected context), but not blue trials (unexpected context). Critically, the predictive nature of the task allowed

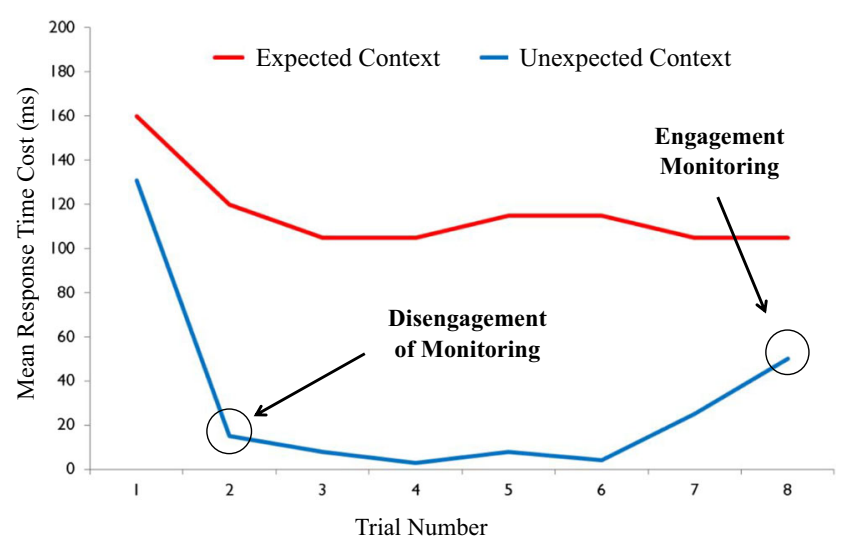

Fig. 1 Graphic representation of the findings reported by Lourenço and Maylor (2014) and the possible cognitive processes that may underlie strategic monitoring across trials. In the expected context, monitoring is generally sustained across trials in preparation for expected prospective memory targets. In contrast, in the unexpected context there is a rapid reduction in cost on Trial 2 that continues across several trials, reflecting the deactivation or disengagement of monitoring. Additionally, there is a preparation, or re-engagement, of monitoring towards the end of the unexpected context in anticipation of the upcoming expected context

participants to anticipate when expected contexts would occur. PM targets were presented in only half of the expected contexts; that is, the PM target was not presented in what we refer to as the expected $_{\mathrm{No}-\mathrm{PM}}$ context. Consequently, monitoring could be compared across trials within contexts that differed only in whether participants were anticipating PM targets (i.e., red trials: expected $_{\text {No-PM }}$ context) or were not anticipating PM targets (i.e., blue trials: unexpected context). As can be seen in Fig. 1, in the expected $_{\mathrm{No}-\mathrm{PM}}$ context there was sustained monitoring as evidenced by cost across all trials, indicative of preparatory engagement of monitoring. In contrast, in the unexpected context there was a substantial reduction in cost on the second trial that was maintained across the middle portions of the context (Trials 2-6). This elimination of cost in the PM block (i.e., response times (RTs) at comparable levels to the control block) is indicative of the deactivation, or disengagement, of monitoring. Additionally, cost increased across the last two trials of the unexpected context (Trials 7-8). This latter finding cannot be explained by bottom-up contextual cueing of task goals, as the stimulus features (color information) were identical to the previous trials in the context. Rather, this slowing is indicative of the top-down preparation, or re-engagement, of monitoring towards the end of the block in anticipation of the upcoming expected block (due to the predictable alternation of context). Together these findings suggest that attentionally demanding processes are needed to flexibly engage and disengage monitoring on a context-by-context and trial-by-trial basis.

Given the attentional demands associated with strategic monitoring (Ball \& Bugg, 2018b; Bugg \& Ball, 2017; Lourenço \& Maylor, 2014), age-related declines in executive attention (Braver \& West, 2008; Hasher \& Zacks, 1988; West, 1996) should presumably result in older adults having greater difficulty 
adjusting monitoring accordingly. The small amount of research on this topic, however, has failed to find support for this idea. Ball and Bugg (2018a) found comparable strategic monitoring patterns between younger and older adults across three experiments. Both age groups were able to reduce monitoring in unexpected contexts when multiple contextual features (e.g., color + location) changed every eight trials and when a single contextual feature (e.g., color) varied randomly on each trial (see Kominsky \& Reese-Melancon, 2017, for similar results using a different type of strategic monitoring procedure). Moreover, neither group was able to reduce monitoring when multiple contextual features (e.g., color + location) varied randomly on each trial. Based on these findings, it was suggested that strategic PM-monitoring processes generally remain intact with increased age. Importantly, however, RTs in those experiments were examined at the aggregate rather than at the trial level. Aggregate level RTs are averaged across all trials within a context (i.e., average of Trials 1-10), whereas at the trial level RTs are calculated separately for each trial within a context (Trial 1, Trial 2, Trial 3, etc.). It is possible that the coarse analyses at the aggregate level may have not been sensitive enough to detect subtle differences in performance between age groups. Therefore, the current study examined RTs at the trial level (similar to Lourenço \& Maylor, 2014) to allow for a more nuanced investigation of the processes involved in strategic monitoring that may be sensitive to agerelated declines in executive attention.

\section{Experiment 1}

The primary aim of Experiment 1 was to examine age differences in the disengagement and re-engagement of monitoring using a predictive (alternating) blocked procedure modeled after Lourenço and Maylor (2014). Participants performed an ongoing lexical decision task in which words and nonwords were presented randomly on each trial during both the control block (no PM intention) and the PM block (with intention). Importantly, stimuli were blocked by color (red or blue) in sets of ten trials and alternated throughout the entire experiment. To examine strategic monitoring, participants were specifically instructed that PM targets ("TOR" syllable) would only occur in red trials. Unbeknownst to participants, PM targets only occurred in only half of the expected contexts. Focusing on the expected contexts in which PM targets did not occur allowed for a direct comparison of monitoring patterns when PM targets

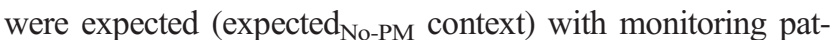
terns when PM targets were not expected (unexpected context). Based on previous research using a similar procedure (Ball \& Bugg, 2018a), we expected that both age groups would be able to reduce monitoring in unexpected contexts relative to expected contexts at the aggregate level. We did, however, anticipate possible age differences to emerge at the trial level.
Lourenço and Maylor (2014) found that monitoring was completely eliminated for younger adults by the second trial and this continued throughout the middle portion of the context (see Fig. 1), indicating the disengagement of monitoring. Given age-related declines in inhibitory functions such as deleting irrelevant information from the focus of attention (Hasher \& Zacks, 1988; Lustig, Hasher, \& Zacks, 2007), older adults may have greater difficulty in disengaging monitoring (i.e., check for "TOR") upon encountering the unexpected context. This could manifest in two possible ways at the trial level. Specifically, older adults may take more time (trials) than young adults to fully disengage from the PM task (i.e., to stop checking for "TOR") in the unexpected block, which would be evidenced by a slower rate of reducing cost across trials for older than for younger adults. Alternatively, older adults may never completely disengage monitoring, which would be evidenced by RTs that are always slower in the PM block than the control block, indicating cost due to possessing an intention that is not necessary for this context (which we refer to as residual cost). Of course, these possibilities are not mutually exclusive. The alternative is that younger and older adults' monitoring patterns are identical, which would be consistent with previous research suggesting that the disengagement of monitoring may not be particularly attentionally demanding (Ball \& Bugg, 2018a).

Assuming monitoring is disengaged during the unexpected context, it needs to be re-engaged to ensure successful target detection in the subsequent expected context. One way to achieve this would be to proactively re-engage monitoring at the end of the unexpected context in anticipation of the upcoming expected context. This appears to be how younger adults in the Lourenço and Maylor (2014) study approached the task, as evidenced by increased cost across the last two trials of the unexpected context. Given age-related deficits in proactive control, such increases in monitoring may not be observed for older adults due to the inability to activate task goals in a preparatory fashion (Bugg, 2014a; Paxton, Barch, Racine, \& Braver, 2008). Instead, older adults may rely on bottom-up contextual features (color or a change in color) to reactively trigger the reengagement of monitoring upon encountering the expected context. Previous research has demonstrated that reactive control generally remains intact with age (e.g., Bugg, 2014b; Shikora, Diede, \& Bugg, 2018). If older adults are more inclined to rely on reactive control, this would be evidenced by no increase in cost on the last trial or two of the unexpected context.

A secondary aim of the current study was to examine a slightly different aspect of strategic monitoring that involves the disengagement of monitoring following successful target detection. To achieve this aim, we instructed participants that PM targets would only occur once within a run of ten trials (e.g., if a target was presented on Trial 5 of the expected context, there would not be another target presented for the next five trials). This allowed for a direct comparison of patterns of monitoring when PM targets were expected but did not appear $\left(\operatorname{expected}_{\text {No-PM }}\right)$ to patterns 
of monitoring when PM targets were expected and did appear and were successfully detected (expected ${ }_{P M}$ ). To our knowledge, no prior study has examined the intentional disengagement of monitoring following target detection (but see Meier \& ReyMermet, 2012, 2018, for related research). However, it is likely that similar inhibitory processes are involved in disengaging monitoring in unexpected contexts (when targets are not expected) and disengaging monitoring following successful detection of targets (when no additional targets are expected). We therefore hypothesized that older adults may again take more time to disengage monitoring (as evidenced by slower rates of reducing cost across trials) or be unable to completely disengage monitoring (as evidenced by residual cost across all trials) compared to younger adults.

\section{Method}

\section{Design and participants}

Thirty younger adults (age 18-23 years) from Washington University received course credit and 29 community-dwelling older adults (age 60-90 years) received monetary compensation for participation. ${ }^{1}$ Only participants reporting normal (or corrected-to-normal) vision and normal color vision were eligible to participate in the study. Sample sizes were based on prior research showing robust effects using similar procedures (Ball \& Bugg, 2018; Bugg \& Ball, 2017; Lourenço et al., 2003; Lourenço \& Maylor, 2014). In particular, Lourenço and Maylor (2014) showed a fairly robust preparatory effect at the end of the unexpected context, which was one of our primary measures of interest. A sample size of 32 per group would be needed to detect a medium-to-large effect ( $d=.65$ ) with a power of .95 , and only 23 to detect a large effect $(d=.80)$. Demographic information can be found in Table 1. All research was approved by the Institutional Review Board at Washington University in St. Louis and was conducted using appropriate ethical guidelines.

\section{Materials}

For the ongoing lexical decision task (LDT), we selected 195 words and 200 nonwords from the ELP database (Balota et al., 2007) that did not include the "TOR" syllable, were four to nine letters in length, and consisted of two to four syllables (mean KF frequency $=40$ ). All items were upper case and appeared in the center of the screen in 30-point red or blue font. Five PM targets were presented during the ongoing task: torch, tortoise, torso, vector, suitor.

\footnotetext{
${ }^{1}$ One additional older adult failed to detect any PM targets during the experiment and was unable to recall the PM instructions in a post-experimental questionnaire. Because this is indicative of a retrospective memory failure rather than a PM failure, this participant was not included in this total or any analyses (Zimmerman \& Meier, 2006).
}

\section{Procedure}

The general procedure for Experiment 1 can be found in Fig. 2 . Participants were first asked to fill out a demographic questionnaire and then given instructions about the ongoing LDT. Participants were told to decide as quickly/accurately as possible whether a string of letters was a word (F key) or a nonword ( $\mathrm{J}$ key). Following each trial, a blank screen appeared and participants pressed the spacebar to progress to the next trial. Participants were then instructed that "the words and nonwords will be presented randomly throughout the ongoing task. However, the color of the items (red, blue) will be blocked in sets of ten trials in an alternating fashion. Thus, you will get ten items presented in red ink, followed by ten items in blue ink, followed by ten items in red ink, and so forth. This ten-item block pattern will continue throughout the experiment." Participants then performed a 30-trial practice LDT phase (ten red, ten blue, ten red) followed by the 200-trial control block (i.e., with no PM instructions). Note, however, that the color alternation was essentially meaningless in the control block since there was no PM intention associated with color at this point.

After the control block participants were given PM instructions. Participants were told that whenever they saw the syllable "TOR" they should press the "7" key after making their lexical decision (or as soon as they remembered). Critically, participants were told that the syllable TOR would only appear in red font (and thus would never appear in blue font). Additionally, participants were instructed that PM targets would only occur once within a run of ten trials of the same context (e.g., "if a PM target occurs on Trial 5, there would not be another PM target for the remaining five trials of that mini-block"). Participants were asked to summarize the instructions, and before continuing the experimenter checked that they understood the target syllable (TOR), the target action (7 key), the context in which targets would appear (red trials), and how many targets would be presented in a mini-block of ten trials (one). Participants then filled out a brief checklist that asked questions about these details before being allowed to move on. A delay of approximately 2.5 min between intention encoding and the PM block was created by having the participants complete the Shipley Vocabulary Test (Shipley, 1940). At the end of the experiment all participants filled out a post-experimental questionnaire to check their memory for the PM instructions (syllable, action, and the color in which targets would appear). All participants remembered this information in both experiments.

The control (200 trials) and PM (200 trials) blocks each consisted of 20 "mini-blocks," with ten red and ten blue mini-block contexts that were presented in alternating fashion throughout the entire experiment. Each mini-block context consisted of ten items (words/nonwords) that were selected randomly on each trial. In the PM block, only five of the ten expected mini-blocks (i.e., red trials) contained PM targets. Therefore, there were five expected ${ }_{\mathrm{PM}}$ contexts and five 
Table 1 Demographic information (Standard Deviation) and Vocabulary Scores for Younger and Older Adults Across Conditions in Experiments 1 and 2

\begin{tabular}{|c|c|c|c|c|}
\hline Experiment & Group & Younger & Older & Significance \\
\hline \multirow[t]{4}{*}{ Experiment 1} & $\mathrm{~N}$ & 30 & 29 & \\
\hline & Age & $18.7(0.91)$ & $69.6(4.79)$ & $*$ \\
\hline & Education & $13.0(0.85)$ & $16.7(2.40)$ & $*$ \\
\hline & Vocabulary & $30.7(3.00)$ & $35.0(2.22)$ & $*$ \\
\hline \multirow[t]{4}{*}{ Experiment 2 (No Counter) } & $\mathrm{N}$ & 35 & 30 & \\
\hline & Age & $19.0(1.15)$ & $68.8(5.96)$ & * \\
\hline & Education & $13.3(1.21)$ & $17.0(2.25)$ & $*$ \\
\hline & Vocabulary & $31.8(2.85)$ & $35.0(3.86)$ & $*$ \\
\hline \multirow[t]{4}{*}{ Experiment 2 (Counter) } & $\mathrm{N}$ & 36 & 30 & \\
\hline & Age & $18.7(.02)$ & $67.4(5.53)$ & $*$ \\
\hline & Education & $12.8(1.11)$ & $16.2(2.17)$ & $*$ \\
\hline & Vocabulary & $32.5(2.74)$ & $35.7(2.66)$ & * \\
\hline
\end{tabular}

Note. Significance column reflects a significant difference across age groups (e.g., older adults had higher vocabulary scores).

expected $_{\text {No-PM }}$ contexts, along with the ten unexpected contexts. The expected ${ }_{\mathrm{PM}}$ contexts occurred in positions $4,8,12$, 16 , and 20 , whereas the expected $\mathrm{No}_{\mathrm{No}}$ contexts appeared in positions $2,6,10,14$, and 18 of the 20 mini-blocks within the overall block (i.e., control or PM). Unexpected contexts occurred in all the odd positions $(1,3,5, \ldots$ etc.). For analytic purposes, we use the terms expected $\mathrm{PM}_{\mathrm{PM}}$, expected $_{\mathrm{No}-\mathrm{PM}}$, and unexpected contexts to refer to the corresponding mini-block position in the control block (e.g., expected ${ }_{\mathrm{PM}}$ contexts were defined as positions 4, 8, 12, 16, and 20 in the control block). In reality, this distinction is arbitrary because there was no PM intention during the control block. PM targets always appeared on the fourth trial of expected $\mathrm{PM}_{\mathrm{PM}}$ contexts $^{2}$, corresponding to trials 34, 74, 114, 154, and 194 of the PM block. The order of PM targets was selected randomly for each participant.

\section{Results}

\section{Data analysis}

Because ongoing task accuracy was high and is often minimally affected by PM task demands (Ball \& Bugg, 2018a; Bugg \& Ball, 2017), we only report full analyses for RTs. For RT analyses, the first five trials of the control and PM blocks, PM target

\footnotetext{
${ }^{2}$ Target presentation was fixed at Trial 4 to allow for the comparison of pretarget and post-target RTs. Ultimately, however, we did not analyze these differences because it is redundant with the comparisons across the two context types $\left(\operatorname{expected}_{\mathrm{PM}}\right.$ and expected $\left.\mathrm{N}_{\mathrm{NoPM}}\right)$. That is, both analyses (pre-target vs. post-target within a context or the same trials between contexts) would demonstrate potential speeding following target detection. Comparing RTs between the two context types on the exact same trials (i.e., 7-10), however, has the advantage of holding all temporal information constant with the only difference between the two context types being target expectancy. In this manner, we believe this is a more sensitive analysis to examine processing differences across context types.
}

trials, the two trials immediately following target presentation (regardless of PM success), and error trials on the LDT were excluded (Ball \& Bugg, 2018a; Bugg \& Ball, 2017). This resulted in the removal of $6 \%$ (younger) and $4 \%$ (older) of the data in the control block and $7 \%$ (younger) and $6 \%$ (older) of the data in the PM block. RTs were trimmed at 3 standard deviations from each participant's mean separately for each context type (e.g., expected ${ }_{\mathrm{PM}}$, expected exo $_{\mathrm{PM}}$, unexpected) and block type (control and PM), resulting in the removal of an additional $2 \%$ of trials within each block for both younger and older adults. Lastly, because slowing always occurred in the PM relative to the control block, analyses are reported on the cost measure (PM block RT - control block RT).

To account for age-related slowing, we transformed RTs to within-participant Z-scores based on the individual's overall mean and standard deviation (Faust, Balota, Spieler, \& Ferraro, 1999). This transformation is necessary because PM demands may have over-additive interactive effects on age given that older adults are starting off at higher levels of the RT scale in the control block, meaning that RT slowing in the PM block may be exaggerated for these individuals. These scaling issues are particularly important in the context of the current study, as we are interested in the rate of change in RTs across trials. Without accounting for baseline differences in RT, age differences in the quadratic reduction in RTs across trials may appear greater for older adults despite the fact that psychologically the two age groups may be performing the task equivalently. Although there are alternative approaches to handle scaling issues such as exGaussian and/or evidence accumulation modeling (Ball et al., 2015; Ball \& Aschenbrenner, 2017), these analytic techniques typically require considerable trial counts to produce reliable parameter estimates. Faust et al. (1999) therefore recommend zscore transformations to examine age differences in RTs. Importantly, because z-score-transformed RT and standard RT 


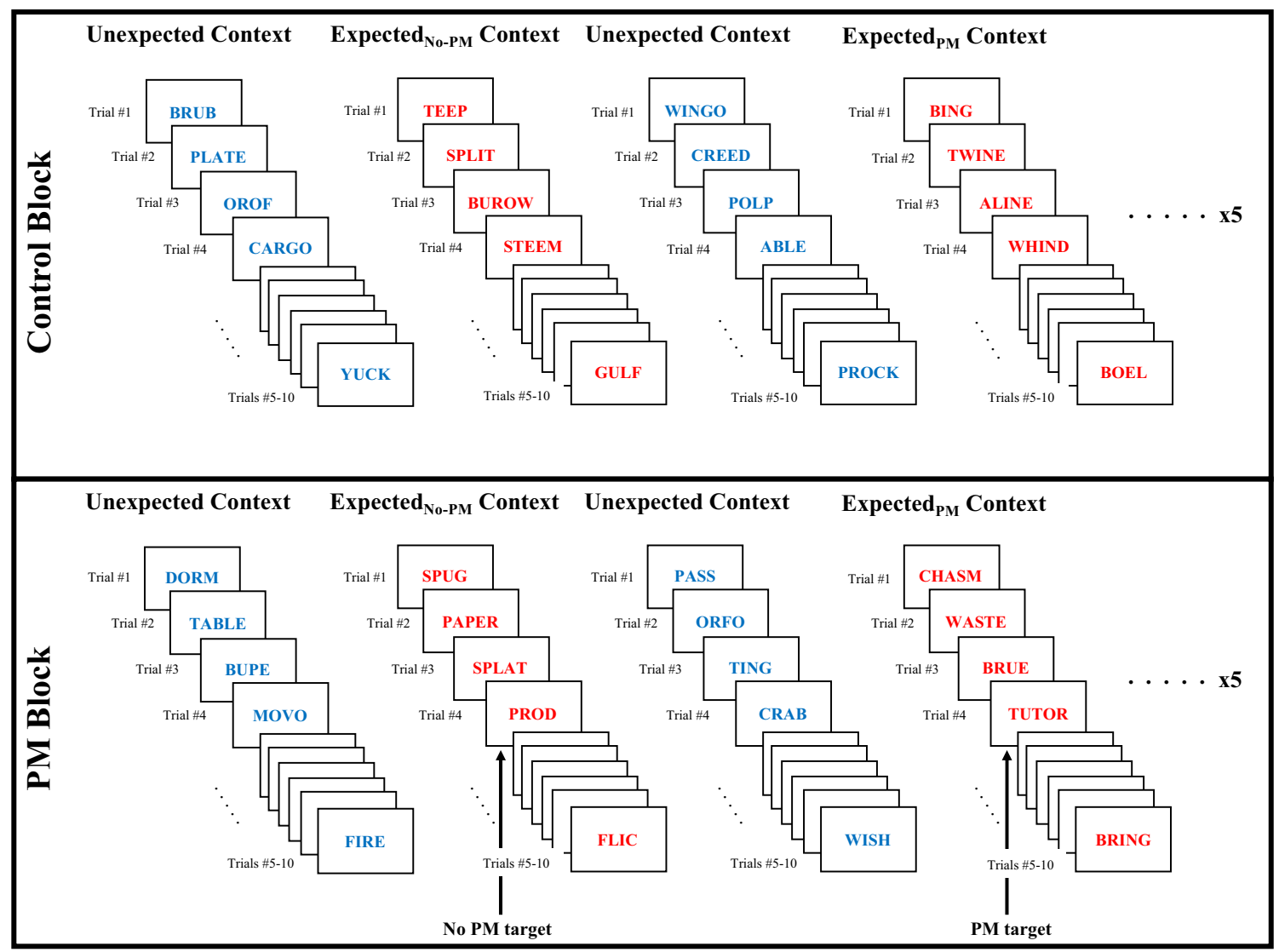

Fig. 2 General procedure for Experiment 1

analyses largely yielded similar results we only report the former here. Complete analyses for standard RTs as well as accuracy can be found in the Supplemental Material. Table 2 displays mean level ongoing task performance in terms of z-score-transformed RTs, standard RTs, and accuracy. Figure 3 presents trial level zscore-transformed RTs separately for each block.

\section{Expected $_{\text {No-PM }}$ versus unexpected}

To examine the disengagement of monitoring when targets were not expected, z-score-transformed RT cost (i.e., PM block z-RT - Control block z-RT) was submitted to a 2 (Age: Younger vs. Older; between-subjects) $\times 2$ (Context Type: Expected $_{\text {No-PM }}$ vs. Unexpected; within-subjects $) \times 10$ (Trial Number: 1-10; within-subjects) mixed-factorial analysis of variance (ANOVA) with planned polynomial contrasts for trial-level effects. Of primary interest for the polynomial contrasts was the quadratic interaction effect with context type or age, which would be indicative of different trajectories of strategic monitoring across contexts or age groups.

Results from the full ANOVA can be found in Table 3. Consistent with previous research, there was evidence of strategic monitoring, with reduced cost in the unexpected relative to the expected $\mathrm{N}_{\mathrm{No}-\mathrm{PM}}$ context (context type: $F=107.91, p<.001$ ). Cost also changed across trials, demonstrating a significant quadratic reduction (trial number: $F=29.35, p<.001$ ). The quadratic reduction in cost across trials differed for the two contexts (trial number $\times$ context type: $F=8.76, p=.004$ ). This interaction reflects a greater quadratic reduction in cost for the unexpected relative to the expected ${ }_{\mathrm{No}-\mathrm{PM}}$ context. As can be seen in the top half of Fig. 4, monitoring was generally sustained across trials in the expected $\mathrm{No}_{\mathrm{N}-\mathrm{PM}}$ context, presumably in preparation for anticipated PM targets (that never occurred). In contrast, and consistent with Lourenço and Maylor (2014), there was a rapid reduction in cost across the first few trials in the unexpected context and this was maintained across the remaining trials. This pattern likely reflects the disengagement of monitoring when targets were not expected. Finally, younger adults showed a marginally larger reduction in cost in the unexpected context relative to the expected $_{\mathrm{No} \text {-PM }}$ context than did older adults, suggesting that older adults tend to show less optimal reductions in monitoring (age $\times$ context: $F=3.89, p=.053$ ). However, there were no other effects of age, indicating comparable rates of strategic disengagement of monitoring across trials between younger and older adults across age groups (all $F$ 's $<1, p$ 's $>.414$ ).

Expected $_{\text {No-PM }}$ versus expected ${ }_{\mathrm{PM}}$ The same general procedure as described above was used to examine z-score-transformed RT cost in the two expected contexts. The difference here is that because we were interested in examining deactivation of 
Table 2 Mean ongoing (standard error) task performance in Experiments 1 and 2

\begin{tabular}{|c|c|c|c|c|c|c|c|c|c|c|c|c|}
\hline Experiment & Condition & Age & DV & Control & PM & Cost & Control & PM & Cost & Control & PM & Cost \\
\hline \multirow[t]{6}{*}{1} & \multirow{6}{*}{ No Counter } & \multirow{3}{*}{ Young } & $\mathrm{z}-\mathrm{RT}$ & $-0.26(0.04)$ & $0.59(0.05)$ & $0.85(0.08)$ & $-0.22(0.03)$ & $0.00(0.04)$ & $0.22(0.07)$ & $-0.20(0.04)$ & $0.22(0.07)$ & $0.42(0.08)$ \\
\hline & & & RT & $692(39$ & $885(42)$ & $193(26)$ & 701 (37) & 744 (34) & $43(18)$ & $692(31)$ & $795(34)$ & $103(20)$ \\
\hline & & & Acc & $0.96(0.01)$ & $0.93(0.01)$ & $-0.03(0.01)$ & $0.94(0.01)$ & $0.94(0.01)$ & $0.00(0.01)$ & $0.94(0.01)$ & $0.93(0.01)$ & $-0.01(0.02)$ \\
\hline & & \multirow[t]{3}{*}{ Old } & z-RT & $-0.26(0.04)$ & $0.53(0.06)$ & $0.79(0.09)$ & $-0.26(0.04)$ & $0.09(0.04)$ & $0.35(0.07)$ & $-0.32(0.04)$ & $0.43(0.08)$ & $0.75(0.11)$ \\
\hline & & & RT & $923(37)$ & 1195 (37) & $272(31)$ & $924(38)$ & $1042(31)$ & 118 (28) & 899 (36) & $1160(47)$ & $260(43)$ \\
\hline & & & Acc & $0.98(0.00)$ & $0.95(0.01)$ & $-0.03(0.01)$ & $0.96(0.01)$ & $0.96(0.01)$ & $0.00(0.01)$ & $0.96(0.01)$ & $0.96(0.01)$ & $0.01(0.01)$ \\
\hline \multirow[t]{12}{*}{2} & \multirow[t]{6}{*}{ No Counter } & \multirow[t]{3}{*}{ Young } & $\mathrm{z}-\mathrm{RT}$ & - & - & - & $-0.12(0.02)$ & $0.05(0.03)$ & $0.17(0.04)$ & $-0.09(0.02)$ & $0.15(0.04)$ & $0.24(0.06)$ \\
\hline & & & RT & - & - & - & 674 (18) & 706 (17) & $32(9)$ & 677 (18) & $731(23)$ & $54(14)$ \\
\hline & & & Acc & - & - & - & $0.94(0.01)$ & $0.95(0.01)$ & $0.01(0.01)$ & $0.95(0.01)$ & $0.95(0.01)$ & $0.00(0.01)$ \\
\hline & & \multirow[t]{3}{*}{ Old } & z-RT & - & - & - & $-0.18(0.03)$ & $0.10(0.04)$ & $0.28(0.06)$ & $-0.18(0.04)$ & $0.33(0.05)$ & $0.51(0.07)$ \\
\hline & & & RT & - & - & - & 851 (26) & $936(35)$ & 85 (19) & $850(28)$ & 989 (39) & $139(24)$ \\
\hline & & & Acc & - & - & - & $0.98(0.00)$ & $0.97(0.01)$ & $-0.01(0.01)$ & $0.97(0.01)$ & $0.97(0.01)$ & $0.00(0.01)$ \\
\hline & \multirow[t]{6}{*}{ Counter } & \multirow[t]{3}{*}{ Young } & z-RT & - & - & - & $-0.07(0.03)$ & $0.01(0.04)$ & $0.08(0.06)$ & $-0.06(0.03)$ & $0.07(0.04)$ & $0.12(0.06)$ \\
\hline & & & RT & - & - & - & 709 (26) & 718 (19) & $9(14)$ & $710(24)$ & $731(20)$ & $21(12)$ \\
\hline & & & Acc & - & - & - & $0.95(0.01)$ & $0.96(0.01)$ & $0.00(0.01)$ & $0.96(0.01)$ & $0.95(0.01)$ & $0.00(0.01)$ \\
\hline & & \multirow[t]{3}{*}{ Old } & z-RT & - & - & - & $-0.21(0.03)$ & $0.14(0.04)$ & $0.35(0.06)$ & $-0.17(0.03)$ & $0.22(0.07)$ & $0.40(0.08)$ \\
\hline & & & RT & - & - & - & 909 (32) & $1026(43)$ & $116(26)$ & 917 (32) & $1045(42)$ & $128(29)$ \\
\hline & & & Acc & - & - & - & $0.98(0.00)$ & $0.97(0.00)$ & $-0.01(0.00)$ & $0.97(0.00)$ & $0.97(0.01)$ & $0.00(0.01)$ \\
\hline
\end{tabular}

Cost is calculated as performance in the PM block minus performance in the control block, meaning that lower values for accuracy actually reflect more cost Note. $z-R T$ z-score-transformed RTs, $R T$ standard response time, Acc accuracy.

monitoring following successful target detection, we only included trials following PM hits ${ }^{3}$ in the expected ${ }_{\mathrm{PM}}$ context (Trials $7-$ 10). For comparison, the corresponding trial numbers (Trials $7-$ 10) were also included from the expected $\mathrm{No}_{\mathrm{No}}$ PM context in the PM block (where monitoring should be sustained since targets never appeared). RTs from the corresponding trials in the control block (Trials 7-10) were included for calculation of cost measures for expected $_{\mathrm{PM}}$ and expected $\mathrm{N}_{\mathrm{No}-\mathrm{PM}}$ contexts, although the distinction between PM and No-PM is arbitrary in the control block since there was no PM intention. Z-transformed RT cost was submitted to a 2 (Age: Younger vs. Older; between-subjects) $\times 2$ (Context Type: Expected $_{\text {No-PM Vs. Expected }}$ PM; within-subjects $) \times 6$ (Trial Number: 7-10; within-subjects) mixed-factorial ANOVA.

There was evidence of strategic monitoring, with reduced cost in the expected $\mathrm{P}_{\mathrm{PM}}$ relative to the expected $\mathrm{No}_{\mathrm{No}}$ context (context type: $F=4.23, p=.044)$. However, this reduction in cost differed between the two age groups (context type $\times$ age: $F=5.57$, $p=.022$ ). As can be seen in the bottom half of Fig. 4 , this interaction reflects that younger, but not older, adults showed reduced cost in the expected ${ }_{\mathrm{PM}}$ relative to the expected $\mathrm{d}_{\mathrm{No} \text {-PM }}$ context. Thus, older adults showed less optimal reductions in monitoring. There were no other significant effects (all $F$ 's $<2.81, p$ 's $>.099$ ).

Residual cost and preparation The previous quadratic contrasts described the time course of strategic disengagement of monitoring across trials. Those results, however, do not speak to whether participants completely disengaged monitoring across trials within the unexpected context and following successful target detection in the expected ${ }_{\mathrm{PM}}$ context, or whether

\footnotetext{
${ }^{3}$ One younger adult remembered the intention but did not detect any targets (i.e., there were no RTs following successful target detection). Instead of excluding this participant, we imputed the missing values based on the sample mean for that age group. This did not significantly alter any results.
}

participants showed evidence of preparatory re-engagement of monitoring at the end of the unexpected context. Replicating the analyses from Lourenco and Maylor (2014), we tested whether z-score-transformed RT cost reliably differed between control and PM blocks on each trial within each context, separately for each age group. Bayesian analyses were used to assess RT differences across blocks using Monte Carlo approximation (100,000 samples) and default diffuse priors in SPSS. Bayes factor estimates less than 0.30 or .10 indicate moderate to strong evidence for the alternative hypothesis, respectively, whereas estimates greater than 3.0 or 10 indicate moderate to strong evidence in favor of the null hypothesis, respectively.

Table 4 displays the results from all post hoc analyses on the z-score-transformed RT cost measures (see also Fig. 4). In summary, both younger and older adults appeared to be monitoring across all trials in the expected $\mathrm{d}_{\mathrm{No}-\mathrm{PM}}$ and expected $\mathrm{PM}_{\mathrm{PM}}$ contexts. The primary difference was that there was moderate evidence that younger adults were generally able to completely disengage monitoring throughout the middle portion (Trials 3-8) of the unexpected context, followed by slowing across the last two trials (although this evidence was weak). In contrast, older adults continued to monitor across all trials of the unexpected context, with only weak evidence that they were able to completely reduce monitoring on Trials 3 and 4 .

\section{Target detection}

The proportion of successfully detected PM targets in the expected block was submitted to a between-subjects ANOVA (left-hand portion of Fig. 5). Consistent with prior research using a similar blocking procedure (Ball \& Bugg, 2018), there was no age difference in performance, $F(1,57)=1.92, \mathrm{p}=.171$, $\eta_{p}{ }^{2}=.033$. 


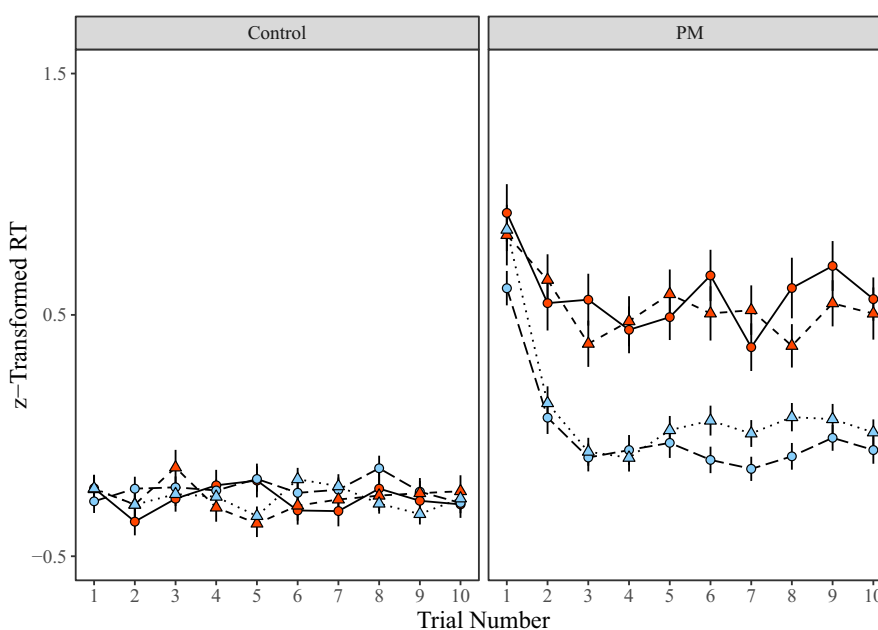

$$
\begin{aligned}
& \text { - Younger: Expected (No-PM) } \\
& -1-\text { Younger: Unexpected } \\
& -\cdots+\text { - - Older: Expected (No-PM) } \\
& \text { …. Older: Unexpected }
\end{aligned}
$$

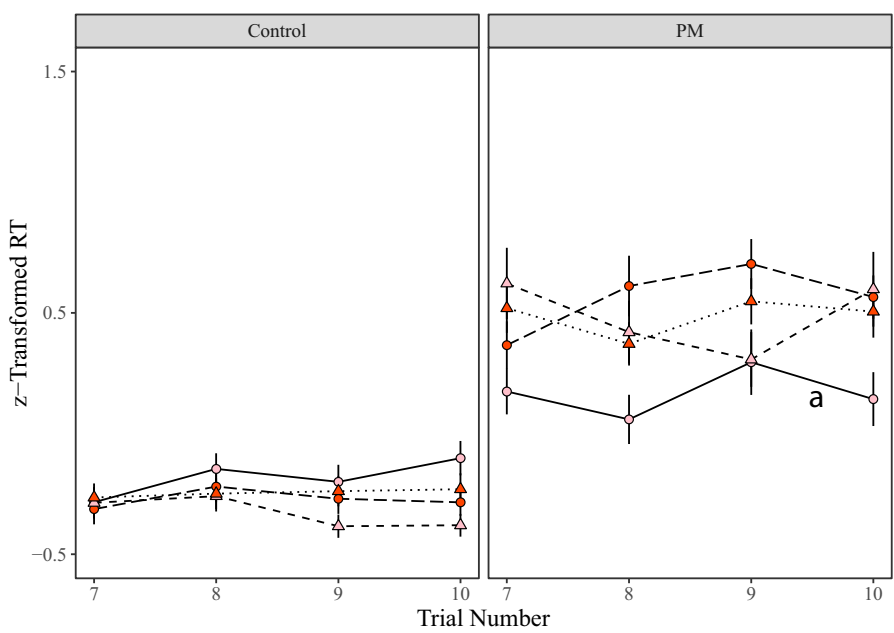

$\longrightarrow$ - Younger: Expected (PM)

- - - - Younger: Expected (No-PM)

- - - - - - Older: Expected (PM)

$\cdots \cdot . \cdot \cdots \cdot$. Older: Expected (No-PM)

Fig. 3 Trial-level z-score-transformed response times (RTs) separately for each block (control and PM) for Experiment 1. Error bars reflect the standard error of the mean

\section{Discussion}

Consistent with prior research (Ball \& Bugg, 2018a), the results of Experiment 1 showed that both younger and older adults were successfully able to reduce monitoring when PM targets were not expected. More interestingly, this experiment demonstrated for the first time that the time course of the monitoring distributions was also comparable at the trial level for both age groups in the unexpected context. There were no significant age effects on the quadratic term, indicating comparable rates of disengaging monitoring across trials in the unexpected context and comparable sustained monitoring across trials in the expected $\mathrm{N}_{\mathrm{No}-\mathrm{PM}}$ context. These findings are consistent with the idea that strategic monitoring processes generally remain intact with increased age (Ball \& Bugg, 2018a; Kominsky \& Reese-Melancon, 2017).

There were, however, some subtle but important age differences that emerged. First, reductions in cost in the unexpected and expected $\mathrm{PM}_{\mathrm{PM}}$ contexts tended to show less differentiation from the expected $\mathrm{N}_{\mathrm{No}-\mathrm{PM}}$ context for older adults than younger adults. Second, while younger adults completely disengaged monitoring across the entire middle portion of the unexpected context (Trials 3 - 8; see also Lourenco \& Maylor, 2014), there was only weak evidence that older adults were able to reduce monitoring, and this was isolated to Trials 3 and 4 . These findings suggest that although strategic monitoring processes generally remain intact with increased age, they may operate less optimally for older adults. In regard to preparatory monitoring, younger adults showed some evidence of slowing on the last two trials of the unexpected context (Lourenço \& Maylor, 2014). Although this effect was relatively small, it is indicative of top-down re-engagement of monitoring in anticipation of the upcoming expected context. For older adults, it is less clear whether they also engaged top-down anticipatory monitoring processes. Although older adults clearly showed a reduction in 
Table 3 Omnibus ANOVA and polynomial contrast results from Experiment 1

\begin{tabular}{|c|c|c|c|c|c|c|c|c|}
\hline ANOVA Context & Variable & Contrast & $d f$ & MSE & $F$ & $h_{p}^{2}$ & $p$ & Significance \\
\hline \multirow[t]{11}{*}{ Expected (No-PM) vs. Unexpected } & Age & & 1,57 & 2.88 & 0.13 & 0.002 & 0.716 & ns \\
\hline & Context Type & & 1,57 & 0.74 & 107.91 & 0.654 & $<.001$ & $*$ \\
\hline & Context Type x Age & & 1,57 & 0.74 & 3.89 & 0.064 & 0.053 & + \\
\hline & Trial Number & Linear & 1,57 & 0.35 & 22.59 & 0.284 & $<.001$ & $*$ \\
\hline & Trial Number & Quadratic & 1,57 & 0.47 & 29.35 & 0.340 & $<.001$ & $*$ \\
\hline & Trial Number x Age & Linear & 1,57 & 0.39 & 0.02 & 0.000 & 0.893 & ns \\
\hline & Trial Number x Age & Quadratic & 1,57 & 0.47 & 0.68 & 0.012 & 0.414 & ns \\
\hline & Trial Number x Context Type & Linear & 1,57 & 0.26 & 5.49 & 0.088 & 0.023 & $*$ \\
\hline & Trial Number x Context Type & Quadratic & 1,57 & 0.33 & 8.76 & 0.133 & 0.004 & $*$ \\
\hline & Trial Number x Context Type x Age & Linear & 1,57 & 0.26 & 0.43 & 0.007 & 0.517 & ns \\
\hline & Trial Number x Context Type x Age & Quadratic & 1,57 & 0.33 & 0.36 & 0.006 & 0.551 & ns \\
\hline \multirow[t]{11}{*}{ Expected (No-PM) vs. Expected (PM) } & Age & & 1,57 & 1.23 & 1.01 & 0.017 & 0.320 & ns \\
\hline & Context Type & & 1,57 & 0.92 & 4.23 & 0.069 & 0.044 & $*$ \\
\hline & Context Type x Age & & 1,57 & 0.92 & 5.57 & 0.089 & 0.022 & $*$ \\
\hline & Trial Number & Linear & 1,57 & 0.50 & 0.30 & 0.005 & 0.583 & ns \\
\hline & Trial Number & Quadratic & 1,57 & 0.50 & 0.11 & 0.002 & 0.742 & ns \\
\hline & Trial Number x Age & Linear & 1,57 & 0.43 & 0.01 & 0.000 & 0.935 & ns \\
\hline & Trial Number x Age & Quadratic & 1,57 & 0.50 & 2.80 & 0.047 & 0.100 & ns \\
\hline & Trial Number x Context Type & Linear & 1,57 & 0.66 & 0.13 & 0.002 & 0.725 & ns \\
\hline & Trial Number x Context Type & Quadratic & 1,57 & 0.49 & 0.99 & 0.017 & 0.323 & ns \\
\hline & Trial Number x Context Type x Age & Linear & 1,57 & 0.67 & 0.32 & 0.006 & 0.576 & ns \\
\hline & Trial Number x Context Type x Age & Quadratic & 1,57 & 0.49 & 0.16 & 0.003 & 0.692 & ns \\
\hline
\end{tabular}

Note. $n s$ not significant, $* \mathrm{p}<.05,+\mathrm{p}<.06$

monitoring across trials of the unexpected context, they continued to show residual cost on all trials. This residual cost could be interpreted as monitoring in anticipation of the upcoming expected context; however, it is also possible this pattern simply reflects less optimal disengagement of monitoring in the unexpected block.

Lastly, there were no differences in target detection across age groups. This is perhaps less surprising given the comparable monitoring patterns between age groups

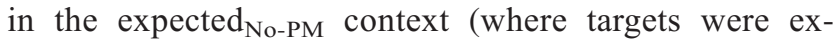
pected) and previous research showing no age differences in target detection using a similar procedure (Ball \& Bugg, 2018a).

\section{Experiment 2}

Experiment 2 was designed as a replication and extension of Experiment 1 to further investigate the subtle age differences in strategic monitoring patterns observed in Experiment 1 (i.e., less optimal disengagement and possibly anticipatory re-engagement of monitoring in unexpected context for older adults). One possible reason that older adults showed little evidence of being able to completely disengage monitoring (i.e., showed no cost) was that they had greater difficulty in tracking where they were within a block of ten trials. For example, older adults may have been disinclined to disengage monitoring in the unexpected context if they had a tough time tracking when the expected context would begin (e.g., for fear of missing targets). Thus, for half of the participants in Experiment 2 a trial counter (110) was presented directly below the ongoing task stimulus to provide environmental support. Previous research has found that younger adults are able to utilize a trial counter to reduce monitoring in unexpected contexts (e.g., Trials 1-24, 31-49, etc.) when instructed that PM targets would only occur on certain trials (e.g., between 25 and 30, 50 and 55, etc.; Bowden, Smith, $\&$ Loft, 2017). We believe that a counter in the current study should reinforce disengagement of monitoring and make it more likely for older adults to completely deactivate monitoring across middle portions of the block (e.g., Trials 3-9) and prepare monitoring later in the block (e.g., Trial 10). This would be evidenced by overall reduced cost and greater rates in the reduction of costs across trials in the counter than the no-counter condition for older adults. 

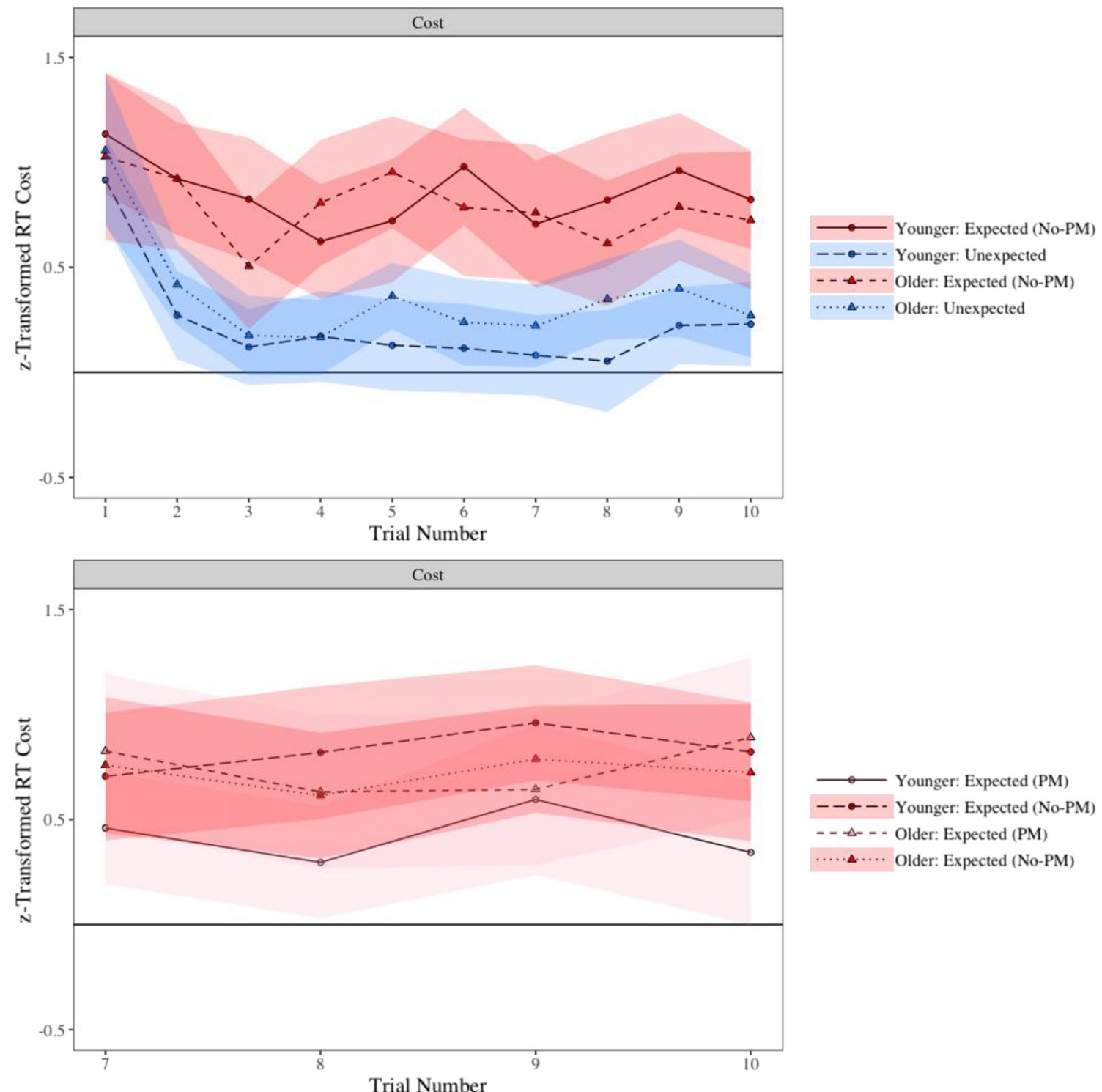

Fig. 4 Trial-level z-score-transformed response-time (RT) cost in Experiment 1 . The upper portion reflects cost in the expected $\mathrm{No}_{\mathrm{No}}$ and unexpected contexts, whereas the bottom portion reflects cost in the

expected $_{\text {No-PM }}$ and expected $d_{P M}$ contexts. Shaded error bars reflect $95 \%$ credible intervals derived from the trial-level block (control vs. PM) analyses

In addition to the counter, another change was made to increase the likelihood of anticipatory monitoring at the end of the unexpected context. In Experiment 1 PM targets were only presented in half of the expected contexts and always occurred several trials into the block (Trial 4). Thus, there was relatively little incentive and possibly a disincentive to engage costly preparatory processes. In Experiment 2, PM targets were presented in every expected context and the targets occurred on the first or second trial of the context. Increased target frequency has been shown to increase preparatory monitoring in expected contexts (Horn \& Bayen, 2015). We reasoned that presenting targets earlier in the expected block (e.g., Trial 1) also should increase preparatory monitoring at the end of the unexpected context to increase the likelihood of detecting PM targets in the subsequent (expected) context. Theoretically, if older adults do not engage preparatory monitoring at the end of the unexpected blocks, they may do worse at detecting targets that are presented on the first trial of the expected block than the second trial.

\section{Method}

\section{Design and participants}

Seventy-one younger adults (age 18-23 years) from Washington University received course credit and 60 community-dwelling older adults (age 60-80 years) 
Table 4 Trial-level analyses examining whether z-transformed RTs differed across blocks in Experiment 1

\begin{tabular}{|c|c|c|c|c|c|c|c|c|c|c|c|}
\hline \multirow[t]{2}{*}{ Context } & \multirow[t]{2}{*}{ Trial } & \multicolumn{5}{|l|}{ Younger } & \multicolumn{5}{|l|}{ Older } \\
\hline & & Mean (SE) & $95 \% \mathrm{CI}$ & $t$ & $p$ & Bayes & Mean (SE) & $95 \% \mathrm{CI}$ & $t$ & $p$ & Bayes \\
\hline Expected $_{P M}$ & 7 & $0.46(0.12)$ & {$[0.19,0.73]$} & 3.69 & 0.001 & 0.03 & $0.83(0.17)$ & {$[0.46,1.19]$} & 4.79 & $<.001$ & $<.001$ \\
\hline Expected $_{P M}$ & 8 & $0.30(0.13)$ & {$[0.03,0.56]$} & 2.37 & 0.025 & 0.58 & $0.63(0.17)$ & {$[0.27,1.00]$} & 3.71 & 0.001 & 0.03 \\
\hline Expected $_{P M}$ & 9 & $0.60(0.17)$ & {$[0.24,0.95]$} & 3.53 & 0.001 & 0.05 & $0.64(0.17)$ & {$[0.28,1.00]$} & 3.82 & 0.001 & 0.02 \\
\hline Expected $_{P M}$ & 10 & $0.34(0.16)$ & {$[0.00,0.69]$} & 2.12 & 0.043 & 0.92 & $0.89(0.18)$ & {$[0.51,1.27]$} & 4.99 & $<.001$ & $<.001$ \\
\hline Expected $_{\mathrm{No}-\mathrm{PM}}$ & 1 & $1.14(0.14)$ & {$[0.85,1.42]$} & 8.40 & $<.001$ & $<.001$ & $1.03(0.19)$ & {$[0.63,1.43]$} & 5.50 & $<.001$ & $<.001$ \\
\hline Expected $_{\mathrm{No}-\mathrm{PM}}$ & 2 & $0.92(0.13)$ & {$[0.65,1.19]$} & 7.29 & $<.001$ & $<.001$ & $0.92(0.16)$ & {$[0.58,1.26]$} & 5.78 & $<.001$ & $<.001$ \\
\hline Expected $_{\text {No-PM }}$ & 3 & $0.83(0.14)$ & {$[0.53,1.12]$} & 5.98 & $<.001$ & $<.001$ & $0.51(0.14)$ & {$[0.21,0.80]$} & 3.61 & 0.001 & 0.04 \\
\hline Expected $_{\text {No-PM }}$ & 4 & $0.62(0.13)$ & {$[0.35,0.90]$} & 4.85 & $<.001$ & $<.001$ & $0.81(0.14)$ & {$[0.51,1.11]$} & 5.73 & $<.001$ & $<.001$ \\
\hline Expected $_{\text {No-PM }}$ & 5 & $0.72(0.14)$ & {$[0.43,1.02]$} & 5.22 & $<.001$ & $<.001$ & $0.95(0.13)$ & {$[0.69,1.22]$} & 7.62 & $<.001$ & $<.001$ \\
\hline Expected $_{\text {No-PM }}$ & 6 & $0.98(0.13)$ & {$[0.70,1.26]$} & 7.44 & $<.001$ & $<.001$ & $0.79(0.15)$ & {$[0.46,1.11]$} & 5.11 & $<.001$ & $<.001$ \\
\hline Expected $_{\text {No-PM }}$ & 7 & $0.71(0.14)$ & {$[0.40,1.01]$} & 4.94 & $<.001$ & $<.001$ & $0.76(0.15)$ & {$[0.44,1.08]$} & 5.01 & $<.001$ & $<.001$ \\
\hline Expected $_{\text {No-PM }}$ & 8 & $0.82(0.15)$ & {$[0.50,1.14]$} & 5.49 & $<.001$ & $<.001$ & $0.61(0.14)$ & {$[0.32,0.91]$} & 4.38 & $<.001$ & 0.01 \\
\hline Expected $_{\text {No-PM }}$ & 9 & $0.96(0.13)$ & {$[0.69,1.24]$} & 7.44 & $<.001$ & $<.001$ & $0.79(0.12)$ & {$[0.53,1.04]$} & 6.57 & $<.001$ & $<.001$ \\
\hline Expected $_{\text {No-PM }}$ & 10 & $0.82(0.11)$ & {$[0.59,1.06]$} & 7.42 & $<.001$ & $<.001$ & $0.72(0.15)$ & {$[0.40,1.05]$} & 4.72 & $<.001$ & $<.001$ \\
\hline Unexpected & 1 & $0.92(0.10)$ & {$[0.71,1.13]$} & 9.33 & $<.001$ & $<.001$ & $1.06(0.17)$ & {$[0.70,1.41]$} & 6.31 & $<.001$ & $<.001$ \\
\hline Unexpected & 2 & $0.27(0.10)$ & {$[0.06,0.48]$} & 2.75 & 0.010 & 0.27 & $0.42(0.09)$ & {$[0.22,0.61]$} & 4.60 & $<.001$ & $<.001$ \\
\hline Unexpected & 3 & $0.12(0.09)$ & {$[-0.06,0.30]$} & 1.41 & 0.169 & 2.77 & $0.18(0.09)$ & {$[-0.01,0.37]$} & 1.98 & 0.058 & 1.17 \\
\hline Unexpected & 4 & $0.17(0.10)$ & {$[-0.05,0.39]$} & 1.68 & 0.103 & 1.90 & $0.17(0.08)$ & {$[-0.01,0.35]$} & 1.99 & 0.056 & 1.15 \\
\hline Unexpected & 5 & $0.13(0.10)$ & {$[-0.09,0.35]$} & 1.26 & 0.216 & 3.31 & $0.36(0.07)$ & {$[0.21,0.52]$} & 4.93 & $<.001$ & $<.001$ \\
\hline Unexpected & 6 & $0.11(0.10)$ & {$[-0.10,0.33]$} & 1.15 & 0.258 & 3.75 & $0.24(0.10)$ & {$[0.03,0.45]$} & 2.46 & 0.020 & 0.48 \\
\hline Unexpected & 7 & $0.08(0.09)$ & {$[-0.11,0.27]$} & 0.90 & 0.376 & 4.79 & $0.22(0.09)$ & {$[0.02,0.42]$} & 2.39 & 0.024 & 0.56 \\
\hline Unexpected & 8 & $0.05(0.11)$ & {$[-0.19,0.30]$} & 0.47 & 0.644 & 6.36 & $0.35(0.09)$ & {$[0.16,0.54]$} & 3.88 & 0.001 & 0.02 \\
\hline Unexpected & 9 & $0.22(0.09)$ & {$[0.04,0.41]$} & 2.58 & 0.015 & 0.38 & $0.40(0.11)$ & {$[0.17,0.63]$} & 3.67 & 0.001 & 0.03 \\
\hline Unexpected & 10 & $0.23(0.09)$ & {$[0.03,0.43]$} & 2.44 & 0.021 & 0.51 & $0.27(0.09)$ & {$[0.07,0.47]$} & 2.89 & 0.007 & 0.20 \\
\hline
\end{tabular}

Note. Bayes factor estimates less than 0.3 or greater than 3.0 indicate moderate evidence in favor of the alternative or null hypothesis, respectively

who did not participate in Experiment 1 received monetary compensation for participation (see Table 1). Only participants reporting normal (or corrected-to-normal) vision and normal color vision were eligible to participate in the study. The effect size for slowing at the end of the unexpected context in Experiment 1 was $d=.433$ and $d=.548$ for younger and older adults, respectively. A sample size of 71 is required to detect an effect of

\section{Experiment 1}

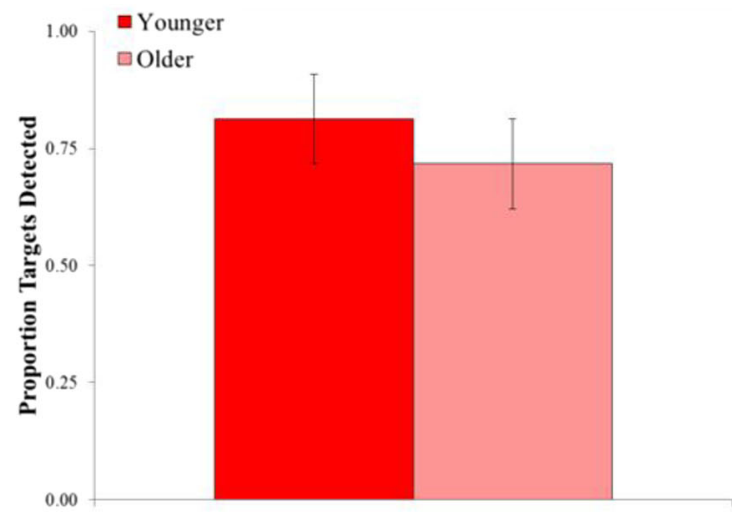

\section{Experiment 2}

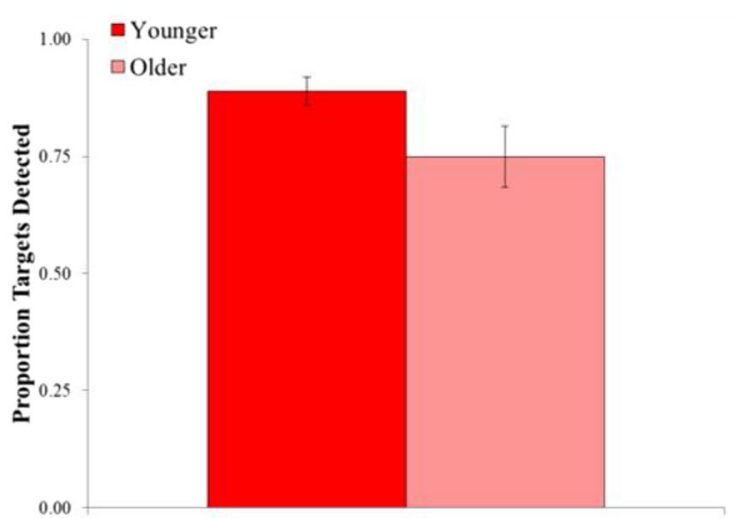

Fig. 5 Target detection for Experiments 1 and 2. Error bars reflect 95\% confidence intervals 
the smallest magnitude with .95 power, and only 46 to detect the larger effect. Thus, our sample size is reasonably within that range.

\section{Procedure}

The procedure (see Fig. 6) was identical to that of Experiment 1 with the following exceptions. First, PM targets occurred in every expected context during the PM block (i.e., block 2, 4, 6, 8 , etc.), whereas they were presented in every other expected context in Experiment 1. This change means that there was no longer an expected $\mathrm{No}_{\mathrm{No} \text {-PM }}$ context in Experiment 2. Secondly, PM targets always appeared on either the first or the second trial of the expected context in alternating fashion (i.e., PM targets occurred on Trial 1 on blocks 2, 6, 10, etc., and on Trial 2 on blocks 4, 8, 12, etc.), whereas targets always appeared on Trial 4 in Experiment 1. The ten PM targets were: torch, tortoise, torso, torn, vector, suitor, motor, torment, actor, and tutor. The order of PM targets was selected randomly for each participant and occurred on trials 11,32, 51, 72, 91, 112, 131, 152, 171, and 192 of the 200 trials in the PM block. Thirdly, for a random half of the participants there was a trial counter placed directly below the string of letters on each trial to specify what trial number the participant was on within the miniblock of ten trials for all phases of the experiment (i.e., practice, control block, PM block). Participants were instructed that the counter would be presented below the stimuli so they could track where they were within the mini-block of ten trials, but were not given any instruction concerning its utility (i.e., participants were not told to use it for increasing or decreasing monitoring). At the end of the experiment participants in the counter condition were asked if they used the counter for any purpose, and if so, what they used it for.

\section{Results}

\section{Data analysis}

The data analytic procedure was identical to Experiment 1. For RT analyses, excluding the first five trials of the control and PM blocks, PM target trials, the two trials immediately following target presentation (regardless of PM success), and error trials on the LDT resulted in the removal of $5 \%$ (younger) and 3\% (older) of the data in the control block and $7 \%$ (younger) and 6\% (older) of the data in the PM block. Trimming RTs at 3 standard deviations resulted in the removal of $2 \%$ of trials within each block for both younger and older adults. Subsequent analyses were performed on z-transformed RT costs. Trial-level z-RTs are displayed in Fig. 7 separately for each block (control vs. PM). Standard RT and accuracy analyses can be found in the Supplemental Material.
Unexpected context To examine the disengagement of monitoring in the unexpected context, z-score-transformed RT cost was submitted to a 2 (Age: Younger vs. Older; between-subjects) $\times 2$ (Condition: No Counter vs. Counter; between-subjects) $\times 10$ (Trial Number: $1-10$; within-subjects) mixedfactorial ANOVA.

Results from the full ANOVA can be found in Table 5 . Consistent with Experiment 1, there was a significant quadratic reduction in cost across trials, indicative of strategic disengagement of monitoring when targets were not expected (trial number: $F=83.83, p<.001)$. Although there was no overall cost difference across conditions (condition: $F<1$ ), there was a significant difference in the quadratic reduction in cost between the two conditions (trial number $\times$ condition: $F=5.92$, $\mathrm{p}=.026$ ). This reflects a larger quadratic reduction in the counter than the no-counter condition. Lastly, as can be seen in the top half of Fig. 8, there was a greater overall cost for older adults, suggesting less optimal reductions in monitoring (age: $F=10.88, \mathrm{p}=.001)$. However, there were no interactions with age, indicating comparable rates of strategic disengagement of monitoring across trials and conditions between younger and older adults (all $F$ 's $<2.18$, $p$ 's $>.142$ ).

Expected $_{\mathrm{PM}}$ context To examine the disengagement of monitoring following target detection, RTs in the expected ${ }_{\mathrm{PM}}$ context were examined on the trials following successful target detection. ${ }^{4}$ Note that for half of the expected contexts PM targets were presented on Trial 1 whereas for the other half PM targets were presented on Trial 2. RTs were examined on Trials 4-9 for the former, and Trials 5-10 for the latter. To equate the two, analyses were performed on trial lag, which denotes the number of trials following target detection $\left(\mathrm{N}_{+3}\right.$, $\left.\mathrm{N}_{+4}, \ldots \mathrm{N}_{+8}\right)$. The corresponding trial numbers $\left(\mathrm{N}_{+3}\right.$ through $\mathrm{N}_{+8}$ ) were included from the control block for the calculation of costs, although again this distinction is arbitrary since there was no PM intention (i.e., no targets) in the control block. Ztransformed RT cost was submitted to a 2 (Age: Younger vs. Older; between-subjects) $\times 2$ (Condition: No Counter vs Counter; between-subjects) $\times 8$ (Trial Lag: $\mathrm{N}_{+3}-\mathrm{N}_{+8}$; within-subjects) mixed-factorial ANOVA.

Results from the full ANOVA can be found in Table 5. There was a significant quadratic reduction in cost across trials, indicative of strategic disengagement of monitoring when no more targets were expected to occur (trial number: $F=6.62$, $p=.011$ ). This reduction in cost did, however, differ as a function of both condition and age (trial number $\times$ condition $\times$ age: $F=4.14, \mathrm{p}=.044)$. This three-way interaction reflects that for younger adults there was a greater quadratic reduction in

\footnotetext{
${ }^{4}$ One older adult remembered the intention but did not detect any targets (i.e., there were no RTs following successful target detection). Instead of excluding this participant, we imputed the missing values based on the sample mean for that age group. This did not significantly alter any results.
} 


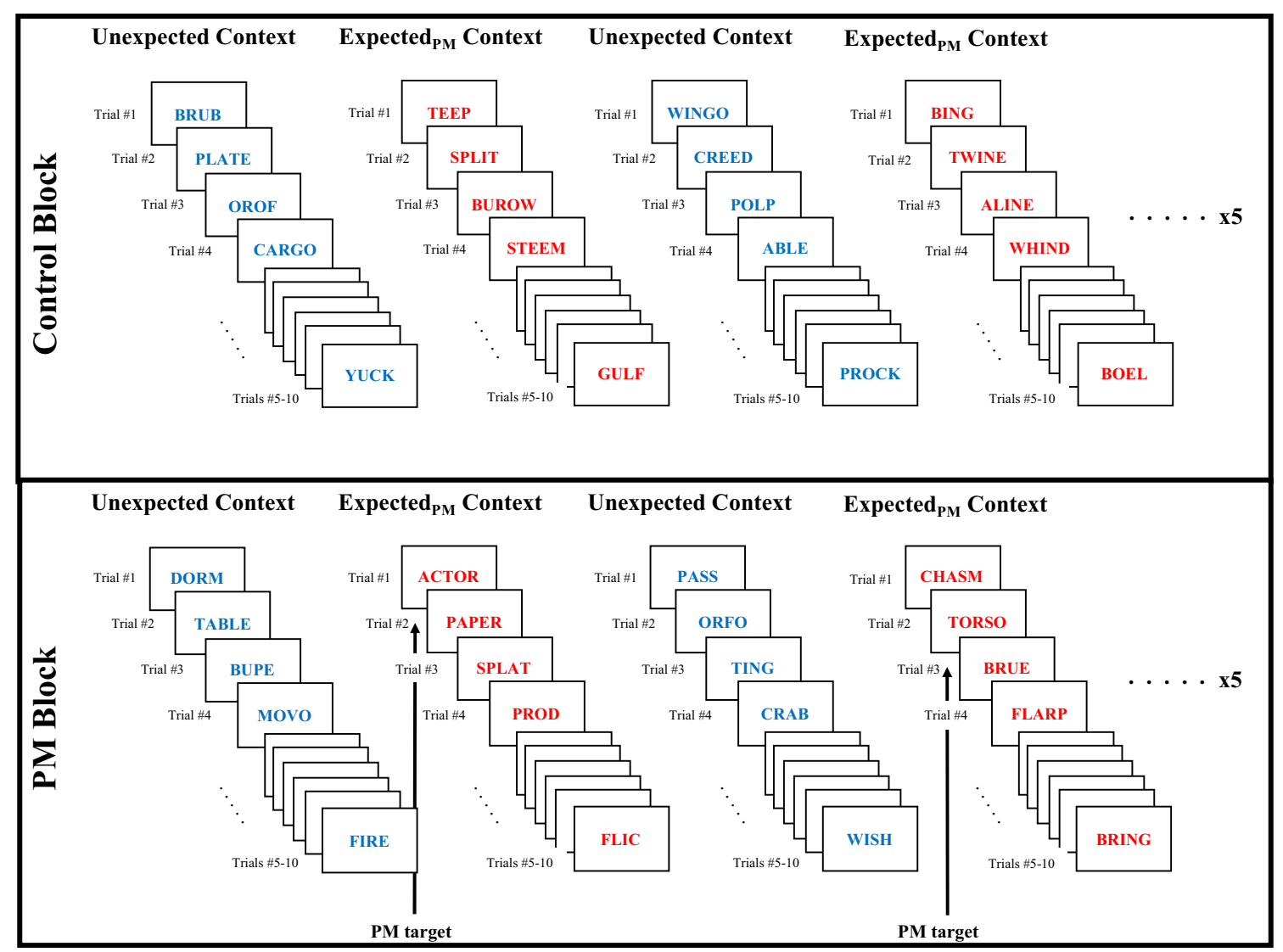

Fig. 6 General procedure for Experiment 2

monitoring in the counter than in the no-counter condition, but there was no difference for older adults. Lastly, there was greater overall cost for older adults, suggesting less optimal reductions in monitoring (age: $F=16.68, \mathrm{p}<.001$ ). There were no other significant effects (all $F$ 's $<3.01, p$ 's $>.085$ ).

Residual cost and preparation To examine whether cost was ever completely eliminated, we tested whether z-scoretransformed RTs reliably differed between control and PM blocks on each trial for each context. The analyses were collapsed across the condition factor since the counter variable had relatively little influence on performance and the power analysis indicated that we were relatively underpowered to separately detect effects across conditions. Bayesian analyses were used to assess RT differences across blocks.

Table 6 displays the results from all post hoc analyses on the z-score-transformed RT cost measures (see also Fig. 8). In summary, older adults appeared to be monitoring across all trials of both contexts. In contrast, there was moderate to strong evidence that younger adults were able to completely disengage monitoring throughout the middle portion (Trials 3-9) of the unexpected context, followed by slowing on the last trial of the unexpected context. Younger adults were also apparently able to reduce monitoring on the fifth trial following target detection in the expected $\mathrm{PM}_{\mathrm{PM}}$ context, but clearly this was not as consistent as the unexpected context.

\section{Target detection}

The proportion of successfully detected PM targets (see righthand portion of Fig. 5) was submitted to a 2 (Trial Number: Trial 1 vs. Trial 2 ; within-subjects) $\times 2$ (Condition: no counter vs. counter; between-subjects) $\times 2$ (Age: younger vs. older; between-subjects) mixed-factorial ANOVA. Contrary to Experiment 1, older adults detected fewer PM targets than younger adults, $F(1,127)=16.41, \mathrm{p}<.001, \eta_{p}{ }^{2}=.114$. Target detection was also marginally better in the counter condition than the no-counter condition, $F(1,127)=3.67, \mathrm{p}=.058$, $\eta_{p}{ }^{2}=.028$. There were no other significant effects, $F$ 's $<1.39$, p's $>.242, \eta_{p}{ }^{2}$ s $<.012$.

\section{Post-experimental questionnaire}

At the end of the experiment participants in the counter condition were asked how (or if) they used the trial counter. As can be seen in Table 7, the counter was mostly used to strategically re-engage monitoring for an upcoming expected context or disengage monitoring following target detection. The 


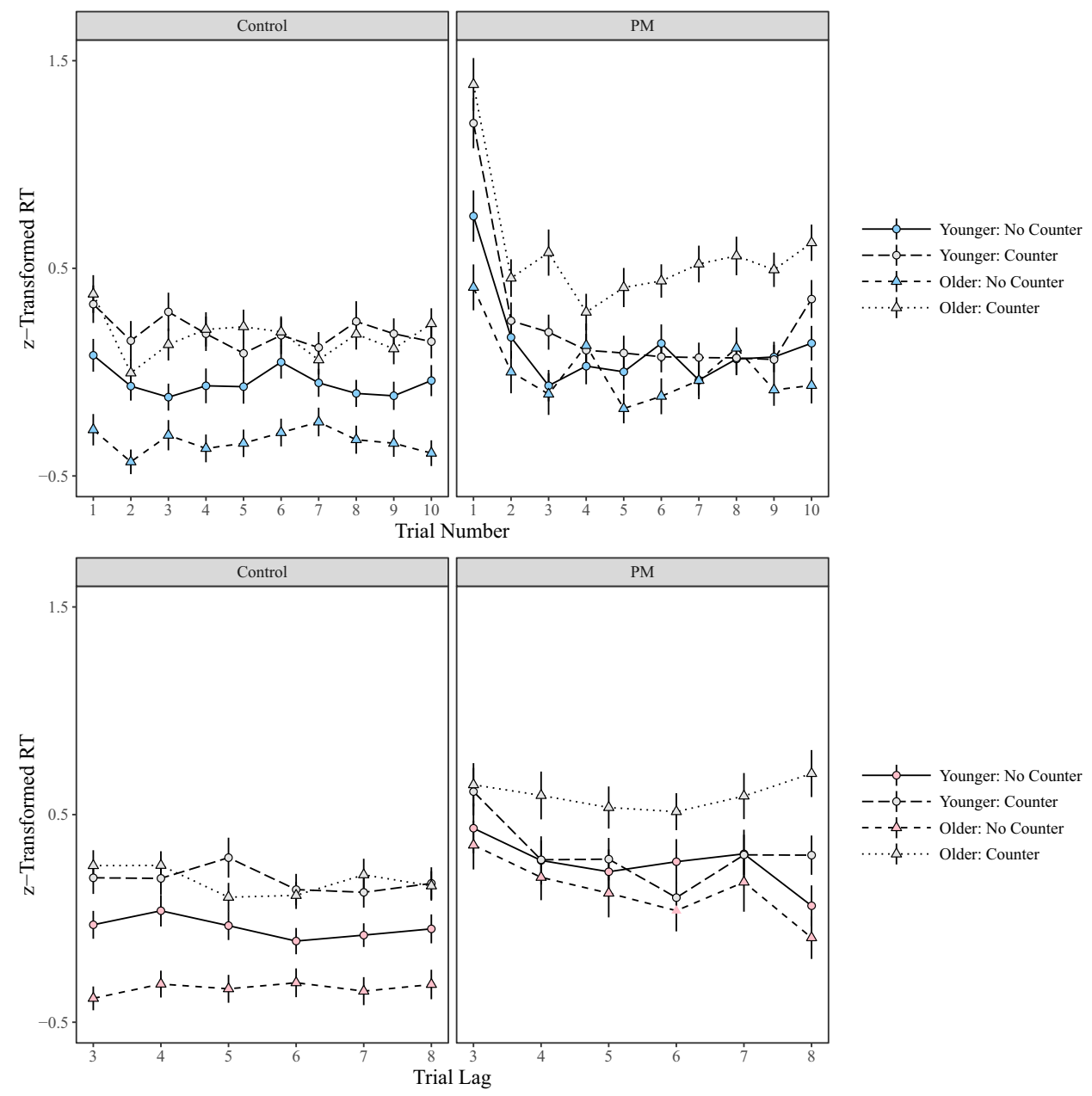

Fig. 7 Trial-level z-score-transformed response times (RTs) separately for each block (control and PM) for Experiment 2. Error bars reflect the standard error of the mean

rationale for not using the counter was primarily that it was not deemed relevant to the task. Critically, more younger adults ${ }^{5}$ reported using the counter than older adults, $\chi^{2}(1$, $\mathrm{N}=66)=15.81, p=.001$. Interestingly, no older adults reported using the counter in a preparatory fashion.

\section{Discussion}

Experiment 2 increased PM target frequency and presented targets earlier in the expected context compared to Experiment 1. Moreover, a trial counter was provided for half of the participants. Despite these changes, the results were largely consistent

\footnotetext{
$\overline{5}$ Exploratory analyses indicated that younger adults who reported using the counter to monitor strategically showed a larger quadratic reduction in cost in the unexpected context than younger adults who did not use the counter. However, no other analyses revealed any differences in counter usage. Exploratory analyses were not conducted with older adults given the small number of participants who reported using the counter.
}

with those of Experiment 1. In particular, at the trial level the rate at which monitoring was disengaged in the unexpected context was identical across age groups. These findings suggest that strategic monitoring processes generally remain intact and operate with comparable temporal efficiency with increased age (Ball \& Bugg, 2018a; Kominsky \& Reese-Melancon, 2017).

As with Experiment 1, however, there were two notable age differences that emerged at the trial level. First, older adults exhibited greater overall cost than younger adults in the unexpected and expected ${ }_{\mathrm{PM}}$ contexts. Second, older adults showed residual cost across all trials of the unexpected context, whereas younger adults completely disengaged monitoring across the entire middle portion (Trials 3-9; see also Lourenco \& Maylor, 2014). These findings again suggest that the strategic disengagement of monitoring may operate less optimally for older adults. As with Experiment 1, younger adults showed slowing on the final trial of the unexpected context, indicative of preparatory monitoring in anticipation of the upcoming expected context. If the residual cost observed across trials for older adults in the unexpected block, 
Table 5 Omnibus ANOVA and polynomial contrast results from Experiment 2

\begin{tabular}{|c|c|c|c|c|c|c|c|c|}
\hline ANOVA Context & Variable & Contrast & $d f$ & $M S E$ & $F$ & $h_{p}^{2}$ & $p$ & Significance \\
\hline \multirow[t]{11}{*}{ Unexpected } & Age & & 1,127 & 1.04 & 10.88 & 0.079 & 0.001 & $*$ \\
\hline & Condition & & 1,127 & 1.06 & 0.02 & 0.000 & 0.900 & ns \\
\hline & Condition $\mathrm{x}$ Age & & 1,127 & 1.04 & 2.17 & 0.017 & 0.143 & ns \\
\hline & Trial Number & Linear & 1,127 & 0.29 & 34.85 & 0.215 & $<.001$ & $*$ \\
\hline & Trial Number & Quadratic & 1,127 & 0.33 & 83.83 & 0.398 & $<.001$ & $*$ \\
\hline & Trial Number x Age & Linear & 1,127 & 0.29 & 1.19 & 0.009 & 0.277 & $\mathrm{~ns}$ \\
\hline & Trial Number x Age & Quadratic & 1,127 & 0.33 & 0.17 & 0.001 & 0.678 & $\mathrm{~ns}$ \\
\hline & Trial Number $\mathrm{x}$ Condition & Linear & 1,127 & 0.29 & 2.15 & 0.017 & 0.145 & $\mathrm{~ns}$ \\
\hline & Trial Number $\mathrm{x}$ Condition & Quadratic & 1,127 & 0.33 & 5.06 & 0.038 & 0.026 & $*$ \\
\hline & Trial Number $\mathrm{x}$ Condition $\mathrm{x}$ Age & Linear & 1,127 & 0.29 & 0.04 & 0.000 & 0.845 & $\mathrm{~ns}$ \\
\hline & Trial Number $\mathrm{x}$ Condition $\mathrm{x}$ Age & Quadratic & 1,127 & 0.33 & 0.63 & 0.005 & 0.428 & $\mathrm{~ns}$ \\
\hline \multirow[t]{11}{*}{ Expected (PM) } & Age & & 1,127 & 0.861 & 6.68 & 0.116 & $<.001$ & $*$ \\
\hline & Condition & & 1,127 & 0.86 & 3.00 & 0.023 & 0.086 & ns \\
\hline & Condition $\mathrm{x}$ Age & & 1,127 & 0.00 & 0.00 & 0.000 & 0.986 & ns \\
\hline & Trial Number & Linear & 1,127 & 0.34 & 4.97 & 0.038 & 0.028 & $*$ \\
\hline & Trial Number & Quadratic & 1,127 & 0.21 & 6.62 & 0.050 & 0.011 & $*$ \\
\hline & Trial Number x Age & Linear & 1,127 & 0.35 & 0.20 & 0.002 & 0.652 & ns \\
\hline & Trial Number x Age & Quadratic & 1,127 & 0.00 & 0.01 & 0.000 & 0.911 & ns \\
\hline & Trial Number x Condition & Linear & 1,127 & 0.34 & 6.86 & 0.051 & 0.010 & $*$ \\
\hline & Trial Number x Condition & Quadratic & 1,127 & 0.21 & 2.15 & 0.017 & 0.145 & ns \\
\hline & Trial Number $\mathrm{x}$ Condition $\mathrm{x}$ Age & Linear & 1,127 & 0.34 & 4.98 & 0.038 & 0.027 & $*$ \\
\hline & Trial Number $\mathrm{x}$ Condition $\mathrm{x}$ Age & Quadratic & 1,127 & 0.21 & 4.14 & 0.032 & 0.044 & $*$ \\
\hline
\end{tabular}

Note. ns not significant, $* \mathrm{p}<.05,+\mathrm{p}<.06$

or especially the residual cost for the final trial or two, reflected preparatory monitoring for older adults, target detection in the (subsequent) expected block presumably should have been comparable across age groups but it was not.

The post-experimental questionnaire provided some interesting insights into possible age differences in strategic monitoring. The counter was mostly used to facilitate strategic reengagement of monitoring for an upcoming expected context or disengagement of monitoring following target detection. Critically, while the majority of younger adults reported using the counter for these purposes, very few older adults did. This suggests that the lack of disengaging/re-engaging of monitoring in the unexpected context for older adults was not simply due to greater difficulty in keeping track of where they were across trials. Rather, older adults appear to have actively avoided using the counter because it was not a critical component of the task (and could even be distracting). Given that older adults did not use the relatively accessible counter to track where they were within a context, it is reasonable to assume that they also did not engage costly resources to actively maintain the trial position to facilitate monitoring in conditions without environmental support. Nevertheless, for a more robust test of preparatory monitoring, future research should consider presenting the counter prior to stimulus onset and explicitly informing participants of the utility of the counter for monitoring and cue detection (e.g., Bowden et al., 2017).

Lastly, in contrast to Experiment 1, older adults detected fewer PM targets than younger adults. Previous research using a similar procedure has shown that target detection is worse for targets presented earlier in the block (e.g., Trial 1) compared to later (e.g., Trial 3; Loft \& Bowden, 2018). It is possible that older adults were relying on reactive control to reengage monitoring upon encountering the expected context, which may be less effective in promoting target detection than engaging proactive control at the end of the unexpected context. It was anticipated that performance may be worse on Trial 1 than Trial 2, but this was not the case.

\section{General discussion}

The current study examined age differences in the engagement and disengagement of monitoring using a blocked procedure in which context varied predictably between expected and unexpected contexts. Across both experiments, younger and older adults successfully utilized contextual information to engage monitoring when contextually appropriate and 

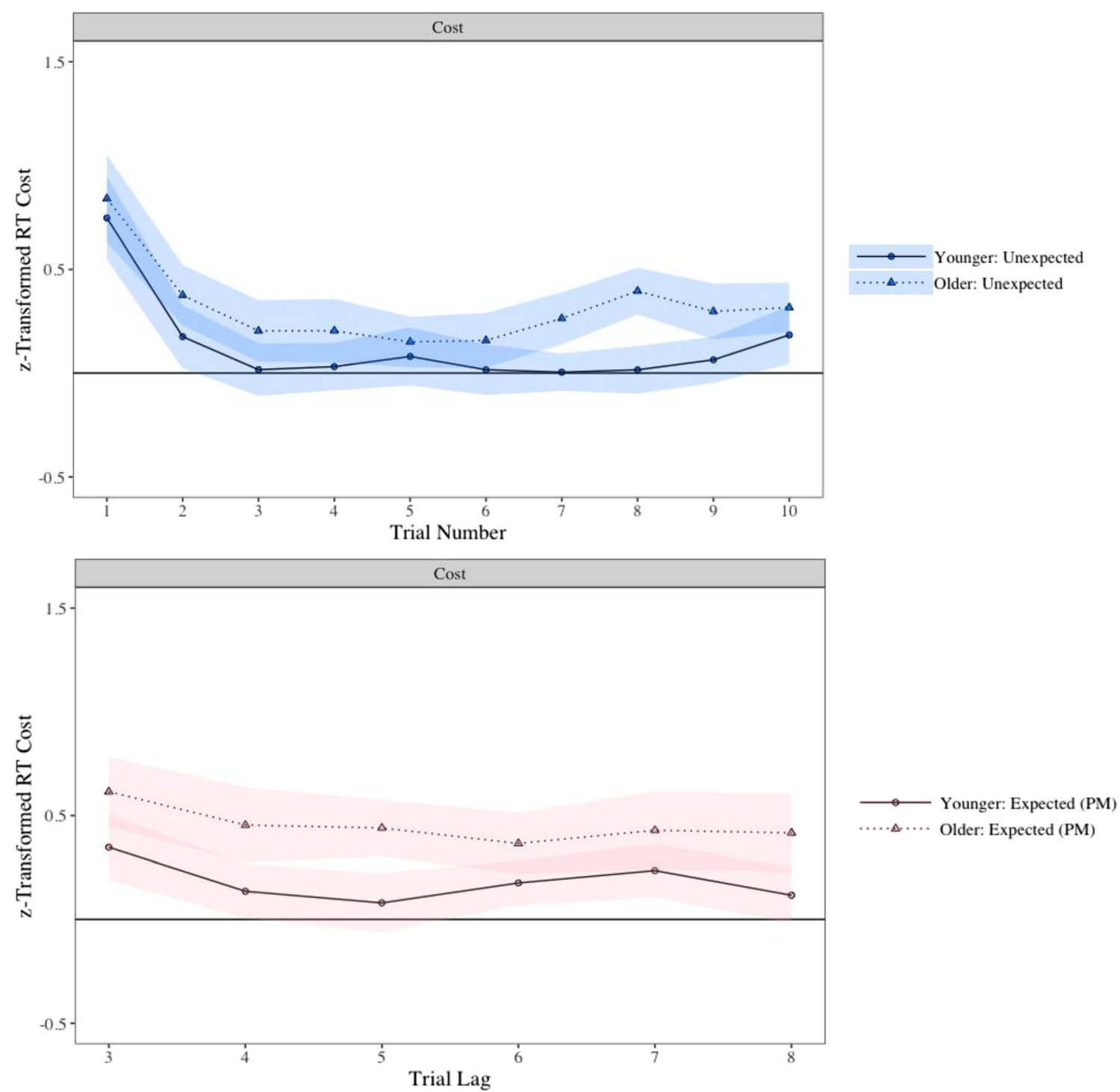

Fig. 8 Trial-level z-score-transformed response time (RT) cost in Experiment 2 (collapsed across condition). The upper portion reflects cost in the unexpected context, whereas the bottom portion reflects cost in the

expected $_{\mathrm{PM}}$ context. Shaded error bars reflect $95 \%$ credible intervals derived from the trial-level block (control vs. PM) analyses

conserve limited-capacity processing resources when contextually inappropriate. Most interestingly, examining the time course of monitoring revealed that the rate with which younger and older adults disengaged monitoring across trials in the unexpected context was comparable. These results add to the growing body of literature suggesting that strategic monitoring ability generally remains intact with increased age across a variety of different contextual features (Ball \& Bugg, 2018a; Kominsky \& Reese-Melancon, 2017) and go a step further in highlighting the temporal specificity with which these processes are enacted. Despite these apparent similarities in monitoring, older adults were generally less effective in eliminating cost in the unexpected context and reducing cost following successful target detection. Below we summarize the similarities and differences between younger and older adults and discuss the theoretical implications of such findings.

\section{Disengagement and re-engagement of monitoring in the unexpected context}

The primary goal of the current study was to examine the strategic disengagement of monitoring in contexts in which targets were not expected. It was hypothesized that age-related declines in inhibitory function (Hasher \& Zacks, 1988; Lustig et al., 2007) may result in older adults taking more time to fully disengage from the PM task (i.e., stop checking for the "TOR" syllable). As can be seen in the change in cost across trials, however, upon encountering the unexpected context 
Table 6 Trial-level analyses examining whether RTs differed across blocks in Experiment 2

\begin{tabular}{|c|c|c|c|c|c|c|c|c|c|c|c|}
\hline \multirow[t]{2}{*}{ Context } & \multirow[t]{2}{*}{ Trial } & \multicolumn{5}{|l|}{ Younger } & \multicolumn{5}{|l|}{ Older } \\
\hline & & Mean (SE) & $95 \% \mathrm{CI}$ & $t$ & $p$ & Bayes & Mean (SE) & $95 \% \mathrm{CI}$ & $t$ & $p$ & Bayes \\
\hline ExpectedPM & $\mathrm{N}+3$ & $0.35(0.08)$ & {$[0.19,0.51]$} & 4.45 & $<.001$ & $<.001$ & $0.62(0.08)$ & {$[0.45,0.78]$} & 7.46 & $<.001$ & $<.001$ \\
\hline ExpectedPM & $\mathrm{N}+4$ & $0.14(0.06)$ & {$[0.00,0.27]$} & 2.09 & 0.040 & 1.33 & $0.45(0.09)$ & {$[0.27,0.64]$} & 5.11 & $<.001$ & $<.001$ \\
\hline ExpectedPM & $\mathrm{N}+5$ & $0.08(0.07)$ & {$[-0.06,0.22]$} & 1.14 & 0.260 & 5.70 & $0.44(0.07)$ & {$[0.30,0.58]$} & 6.54 & $<.001$ & $<.001$ \\
\hline ExpectedPM & $\mathrm{N}+6$ & $0.18(0.05)$ & {$[0.07,0.28]$} & 3.32 & 0.001 & 0.07 & $0.37(0.07)$ & {$[0.22,0.51]$} & 5.05 & $<.001$ & $<.001$ \\
\hline ExpectedPM & $\mathrm{N}+7$ & $0.24(0.06)$ & {$[0.11,0.36]$} & 3.69 & $<.001$ & 0.02 & $0.43(0.09)$ & {$[0.24,0.62]$} & 4.64 & $<.001$ & $<.001$ \\
\hline ExpectedPM & $\mathrm{N}+8$ & $0.12(0.07)$ & {$[-0.02,0.25]$} & 1.75 & 0.085 & 2.45 & $0.42(0.09)$ & {$[0.23,0.61]$} & 4.45 & $<.001$ & $<.001$ \\
\hline Unexpected & 1 & $0.75(0.10)$ & {$[0.55,0.95]$} & 7.56 & $<.001$ & $<.001$ & $0.84(0.10)$ & {$[0.63,1.05]$} & 8.13 & $<.001$ & $<.001$ \\
\hline Unexpected & 2 & $0.18(0.08)$ & {$[0.02,0.33]$} & 2.34 & 0.022 & 0.80 & $0.38(0.07)$ & {$[0.23,0.52]$} & 5.25 & $<.001$ & $<.001$ \\
\hline Unexpected & 3 & $0.02(0.06)$ & {$[-0.11,0.14]$} & 0.26 & 0.794 & 10.37 & $0.20(0.07)$ & {$[0.06,0.35]$} & 2.79 & 0.007 & 0.27 \\
\hline Unexpected & 4 & $0.03(0.06)$ & {$[-0.08,0.15]$} & 0.55 & 0.585 & 9.25 & $0.20(0.08)$ & {$[0.05,0.36]$} & 2.70 & 0.009 & 0.33 \\
\hline Unexpected & 5 & $0.08(0.07)$ & {$[-0.06,0.22]$} & 1.16 & 0.249 & 5.54 & $0.15(0.06)$ & {$[0.03,0.27]$} & 2.52 & 0.014 & 0.50 \\
\hline Unexpected & 6 & $0.02(0.06)$ & {$[-0.11,0.14]$} & 0.27 & 0.791 & 10.36 & $0.16(0.07)$ & {$[0.03,0.29]$} & 2.42 & 0.018 & 0.62 \\
\hline Unexpected & 7 & $0.00(0.04)$ & {$[-0.09,0.09]$} & 0.09 & 0.928 & 10.69 & $0.26(0.06)$ & {$[0.14,0.39]$} & 4.28 & $<.001$ & $<.001$ \\
\hline Unexpected & 8 & $0.02(0.06)$ & {$[-0.10,0.13]$} & 0.28 & 0.783 & 10.33 & $0.40(0.06)$ & {$[0.28,0.51]$} & 7.14 & $<.001$ & $<.001$ \\
\hline Unexpected & 9 & $0.06(0.06)$ & {$[-0.05,0.18]$} & 1.16 & 0.251 & 5.57 & $0.30(0.07)$ & {$[0.16,0.43]$} & 4.51 & $<.001$ & $<.001$ \\
\hline Unexpected & 10 & $0.18(0.07)$ & {$[0.04,0.32]$} & 2.67 & 0.009 & 0.37 & $0.32(0.06)$ & {$[0.20,0.44]$} & 5.41 & $<.001$ & $<.001$ \\
\hline
\end{tabular}

Note. Bayes factor estimates less than 0.3 or greater than 3.0 indicate moderate evidence in favor of the alternative or null hypothesis, respectively

both age groups were quickly able to reduce monitoring and maintained this disengagement across trials. The comparable monitoring patterns between younger and older adults could reflect that disengaging monitoring might not be particularly attentionally demanding in comparison to the engagement of monitoring (i.e., checking for TOR). Alternatively, contextual features (i.e., the color blue) may have reactively triggered task goals (i.e., "do not check for TOR"), thereby placing relatively minimal demands on inhibitory functioning (see also Ball \& Bugg, 2018a). In any manner, these results clearly demonstrate that attentional declines that typically accompany aging do not appear to influence the temporal efficiency with which strategic disengagement of monitoring occurs.

The primary difference between age groups was that in the unexpected context older adults tended to show less differentiation in the reduction of cost relative to the expected $\mathrm{N}_{\mathrm{No}-\mathrm{PM}}$ context (Experiment 1), had greater overall cost (Experiment 2), and had residual cost for the majority of trials (Trials $5-10$ in Experiment 1 and all trials in Experiment 2). These findings suggest that although the temporal efficiency of disengaging monitoring is similar between age groups, the optimality, or "completeness," of this disengagement might differ. This

Table 7 Self-reported counter usage in the counter condition of Experiment 2

\begin{tabular}{|c|c|c|c|c|}
\hline \multirow[t]{2}{*}{ Counter Usage } & \multicolumn{2}{|l|}{ Younger } & \multicolumn{2}{|l|}{ Older } \\
\hline & $N$ & $\%$ & $N$ & $\%$ \\
\hline \multicolumn{5}{|l|}{ Overall } \\
\hline Claimed to use counter & 26 of 36 & 0.72 & 6 of 30 & 0.20 \\
\hline \multicolumn{5}{|l|}{ Did Use Counter } \\
\hline \multicolumn{5}{|l|}{ General Tracking: } \\
\hline Track how many trials were left in the context & 4 of 26 & 0.15 & 2 of 6 & 0.33 \\
\hline \multicolumn{5}{|l|}{ Preparatory Re-Engagment in Blue Context: } \\
\hline Prepared to look for TOR at end of blue context & 12 of 26 & 0.46 & 0 of 6 & 0.00 \\
\hline \multicolumn{5}{|l|}{ Disengagment of Monitoring in Red Context: } \\
\hline Reduce monitoring after target detection in red context & 12 of 26 & 0.46 & 4 of 6 & 0.67 \\
\hline
\end{tabular}


could in part reflect greater variability in monitoring for older adults, with greater age-related lapses of attention or intention (West, Murphy, Armilio, Craik, \& Stuss, 2002). That is, while older adults were generally successfully able to disengage monitoring, there may have been a greater subset of trials in which attentional lapses resulted in disengagement failures. This could have been exacerbated due to the relatively high demands placed on working memory (Verhaeghen et al., 2003) due to participants needing to maintain multiple pieces of information (e.g., intended action, PM target, context information, trial counter).

Alternatively, age-related residual slowing could be strategic or adaptive in nature. By maintaining task-irrelevant goals (i.e., check for TOR) at an above-threshold level of activation in the unexpected context, the need to engage self-initiated retrieval processes upon encountering the expected context would be reduced or eliminated. Guynn (2003) has described this as the maintenance of a prospective retrieval mode, which reflects a state of global readiness to treat incoming stimuli as potential retrieval cues associated with the intended action. Older adults may have opted to rely on a memory maintenance process to reduce reliance on memory retrieval (Touron \& Hertzog, 2004), or may have simply been more cautious in their responding (Heathcote et al., 2015; Starns \& Ratcliff, 2010) to reduce the likelihood of missing PM targets. More work is needed to better specify the exact mechanisms underlying this residual cost for older adults, as it is important to understand how to reduce unnecessary engagement of costly monitoring resources in contexts in which targets are not expected to appear. ${ }^{6}$ Future research fitting evidenceaccumulation models to the observed data might better disentangle these theoretical alternatives, as these models are associated with specific cognitive processes (e.g., response caution, speed of processing, variability) that may be useful for understanding age differences in strategic monitoring ability (Ball \& Aschenbrenner, 2017; Horn, Bayen, \& Smith, 2013; Strickland et al., 2018; Strickland, Elliot, Wilson, Loft, Neal, $\&$ Heathcote, 2019).

With regard to the preparatory re-engagement of monitoring, only younger adults clearly showed evidence of an increase in monitoring on the last trial of the unexpected context. As noted by Lourenço and Maylor (2014), such findings cannot be explained by bottom-up contextual cueing of task goals (i.e., reactive control), as the

\footnotetext{
${ }^{6}$ One remaining possibility is that the residual cost for older adults may reflect that they were differentially impacted by practice (or fatigue) effects given that the PM block always followed the control block (see also Ball \& Bugg, 2018; Bugg \& Ball, 2017; Lourenço \& Maylor, 2014). To address this concern, we compared performance between the first and second half of each block (control and PM). Importantly, while participants did get faster over time, these practice effects did not appear to differentially influence performance for younger and older adults. That being said, a more optimal design choice for future research would be to counterbalance block type to ensure that fatigue or practice effects do not influence performance.
}

stimulus features (color information) were identical to the previous trials in the block. Rather, these findings suggest that younger adults were engaging top-down, preparatory monitoring in anticipation for the upcoming expected context. In contrast, older adults may have relied on less attentionally demanding reactive control processes to re-engage monitoring upon encountering the expected context. The alternative is that older adults may have remained in a heightened preparatory state throughout the entire unexpected context, possibly reflecting the maintenance of a prospective retrieval mode. While we cannot completely rule out this interpretation, presumably target detection should have been comparable between younger and older adults in Experiment 2 if this was the case. A possible reconciliation of both views is that older participants indeed maintained a prospective retrieval mode in a preparatory manner, but nonetheless failed at checking for PM targets (Ball \& Aschenbrenner, 2017). That is, the cost to ongoing task performance seen across the majority of trials may not have been functional for target detection. Future research should aim to find ways to eliminate monitoring across trials for older adults (e.g., playing a tone on Trial 9 rather than using a counter) to allow for a stronger test of preparatory monitoring at the end of the unexpected context.

\section{Disengagement of monitoring in the $\operatorname{expected}_{\mathrm{PM}}$ context}

A secondary aim of the current study was to examine for the first time the strategic disengagement of monitoring following successful target detection in the expected $\mathrm{PM}_{\mathrm{PM}}$ context. While younger adults were generally able to reduce monitoring following target detection, older adults showed no reduction in monitoring relative to the expected ${ }_{\mathrm{No}-\mathrm{PM}}$ context (Experiment 1) or greater overall cost (Experiment 2). It is possible that bottom-up perceptual features (the color red) may have reinforced maintenance of the prospective retrieval mode that produced cost despite older adults knowing that targets would not occur again. Notably, even for younger adults residual cost was evident across the majority of trials in both experiments. These findings suggest that perhaps not all context is utilized equally and completely disengaging monitoring in expected contexts may be more difficult than doing so in unexpected contexts. An interesting avenue for future research would be to inform participants that the intention is finished (e.g., "you no longer have to respond to PM targets"; Scullin \& Bugg, 2013) and examine whether age differences emerge in residual cost or false alarms to PM targets in the context previously associated with PM responding (i.e., red trials). This could have important applied implications for how contextual features may trigger inappropriate monitoring or retrieval processes. 


\section{Conclusion}

The findings from the current study are both theoretically and practically important. Theoretically, the results suggest that although strategic monitoring generally remains intact with increased age, these processes operate less optimally for older adults. Practically, the finding that older adults continue to show residual cost across the majority of trials of the unexpected context suggests that older adults may have greater disruptions to daily activities because of unnecessary engagement of costly monitoring resources in contexts in which targets are not expected to appear. Additionally, the lack of preparatory monitoring in anticipation of encountering a context in which targets are expected to appear may ultimately result in poorer intention fulfillment under certain scenarios. Future research aimed at increasing the efficacy of disengagement and preparatory re-engagement of monitoring with increased age is therefore of critical importance for promoting healthy aging.

Acknowledgements B. Hunter Ball was supported by an NIA Training Grant (T32AG000030-40) at Washington University during data collection and writing of portions of this article. We would like to extend a special acknowledgment to Brigida Rusconi and Jihyun Suh for their assistance in previous versions of the manuscript. We also thank Kierra Harris, Christina Rao, and Erica Williams for their assistance in data collection and entry.

Data Availability The data and materials for all experiments are available upon request. The experiments were not preregistered. Portions of the data were presented at the biennial Cognitive Aging Conference in Atlanta, GA.

\section{References}

Ball, B. H., \& Aschenbrenner, A. J. (2017). The importance of age-related differences in prospective memory: Evidence from diffusion model analyses. Psychonomic Bulletin \& Review, 25(3), 1114-1122.

Ball, B. H., \& Brewer, G. A. (2018). Proactive control processes in eventbased prospective memory: Evidence from intraindividual variability and ex-Gaussian analyses. Journal of Experimental Psychology: Learning, Memory, \& Cognition, 44(5), 793.

Ball, B. H., Brewer, G. A., Loft, S., \& Bowden, V. (2015). Uncovering continuous and transient monitoring profiles in event-based prospective memory. Psychonomic Bulletin \& Review, 22(2), 492-499.

Ball, B. H., \& Bugg, J. M. (2018). Aging and the strategic use of context to control prospective memory monitoring. Psychology and Aging, 33(3), 527.

Ball, B. H., \& Bugg, J. M. (2018a). Context cue focality influences strategic prospective memory monitoring. Psychonomic Bulletin \& Review, 25(4), 1405-1415.

Balota, D. A., Yap, M. J., Hutchison, K. A., Cortese, M. J., Kessler, B., Loftis, B., ... \& Treiman, R. (2007). The English lexicon project. Behavior Research Methods, 39(3), 445-459.

Bowden, V. K., Smith, R. E., \& Loft, S. (2017). Eye movements provide insights into the conscious use of context in prospective memory. Consciousness and Cognition, 52, 68-74.

Braver, T. S., \& West, R. (2008). Working memory, executive control, and aging. The Handbook of Aging and Cognition, 3, 311-372.
Bugg, J. M. (2014a). Conflict-triggered top-down control: Default mode, last resort, or no such thing? Journal of Experimental Psychology: Learning, Memory, and Cognition, 40, 567-587.

Bugg, J. M. (2014b). Evidence for the sparing of reactive cognitive control with age. Psychology and Aging, 29(1), 115.

Bugg, J. M., \& Ball, B. H. (2017). The strategic control of prospective memory monitoring in response to complex and probabilistic contextual cues. Memory \& Cognition, 1-21.

Cohen, A. L., Jaudas, A., Hirschhorn, E., Sobin, Y., \& Gollwitzer, P. M. (2012). The specificity of prospective memory costs. Memory, 20(8), 848-864.

Faust, M. E., Balota, D. A., Spieler, D. H., \& Ferraro, F. R. (1999). Individual differences in information-processing rate and amount: implications for group differences in response latency. Psychological Bulletin, 125(6), 777.

Guynn, M.J. (2003). A two-process model of strategic monitoring in event-based prospective memory: Activation/retrieval mode and checking. International Journal of Psychology, 38, 245-256.

Hasher, L., \& Zacks, R. T. (1988). Working memory, comprehension, and aging: A review and a new view. Psychology of Learning and Motivation, 22, 193-225.

Heathcote, A., Loft, S., \& Remington, R. W. (2015). Slow down and remember to remember! A delay theory of prospective memory costs. Psychological Review, 122(2), 376.

Horn, S. S., \& Bayen, U. J. (2015). Modeling criterion shifts and target checking in prospective memory monitoring. Journal of Experimental Psychology: Learning, Memory, and Cognition, 41(1), 95.

Horn, S. S., Bayen, U. J., \& Smith, R. E. (2013). Adult age differences in interference from a prospective-memory task: A diffusion model analysis. Psychonomic Bulletin \& Review, 20(6), 1266-1273.

Kliegel, M., Jäger, T., \& Phillips, L. H. (2008). Adult age differences in event-based prospective memory: A meta-analysis on the role of focal versus nonfocal cues. Psychol Aging, 23, 203-208.

Knight, J. B., Meeks, J. T., Marsh, R. L., Cook, G. I., Brewer, G. A., \& Hicks, J. L. (2011). An observation on the spontaneous noticing of prospective memory event-based cues. Journal of Experimental Psychology: Learning, Memory, and Cognition, 37(2), 298.

Kominsky, T. K., \& Reese-Melancon, C. (2017). Effects of context expectation on prospective memory performance among older and younger adults. Memory, 25(1), 122-131.

Kuhlmann, B. G., \& Rummel, J. (2014). Context-specific prospectivememory processing: Evidence for flexible attention allocation adjustments after intention encoding. Memory \& Cognition, 42(6), 943-949.

Lourenço, J. S., \& Maylor, E. A. (2014). Is it relevant? Influence of trial manipulations of prospective memory context on task interference. The Quarterly Journal of Experimental Psychology, 67(4), 687-702.

Lourenço, J. S., White, K., \& Maylor, E. A. (2013). Target context specification can reduce costs in nonfocal prospective memory. Journal of Experimental Psychology: Learning, Memory, and Cognition, 39(6), 1757.

Lustig, C., Hasher, L., \& Zacks, R. T. (2007). Inhibitory deficit theory: Recent developments in a "new view". Inhibition in cognition, 17, 145-162.

Marsh, R. L., Hicks, J. L., \& Cook, G. I. (2006). Task interference from prospective memories covaries with contextual associations of fulfilling them. Memory \& Cognition, 34, 1037-1045.

Marsh, R. L., Hicks, J. L., Cook, G. I., Hansen, J. S., \& Pallos, A. L. (2003). Interference to ongoing activities covaries with the characteristics of an event-based intention. Journal of Experimental Psychology: Learning, Memory, and Cognition, 29(5), 861.

Meier, B., \& Rey-Mermet, A. (2012). Beyond monitoring: After-effects of responding to prospective memory targets. Consciousness and Cognition, 21(4), 1644-1653. 
Meier, B., \& Rey-Mermet, A. (2018). After-effects without monitoring costs: The impact of prospective memory instructions on task switching performance. Acta Psychologica, 184, 85-99.

Paxton, J. L., Barch, D. M., Racine, C. A., \& Braver, T. S. (2008). Cognitive control, goal maintenance, and prefrontal function in healthy aging. Cerebral Cortex, 18(5), 1010-1028.

Rendell, P. G., McDaniel, M. A., Forbes, R. D., \& Einstein, G. O. (2007). Age-related effects in prospective memory are modulated by ongoing task complexity and relation to target cue. Aging, Neuropsychology, and Cognition, 14, 236-256.

Scullin, M. K., \& Bugg, J. M. (2013). Failing to forget: Prospective memory commission errors can result from spontaneous retrieval and impaired executive control. Journal of Experimental Psychology: Learning, Memory, and Cognition, 39(3), 965.

Shipley, W. C. (1940). A self-administering scale for measuring intellectual impairment and deterioration. The Journal of Psychology, 9, $371-377$.

Smith, R.E. (2003). The cost of remembering to remember in event-based prospective memory: Investigating the capacity demands of delayed intention performance. Journal of Experimental Psychology: Learning, Memory, \& Cognition, 29, 347-361.

Smith, R. E. (2016). Prospective Memory in Context. Psychology of Learning and Motivation, 66, 211-249.

Smith, R. E., Hunt, R. R., McVay, J. C., \& McConnell, M. D. (2007). The cost of event-based prospective memory: salient target events. Journal of Experimental Psychology: Learning, Memory, and Cognition, 33(4), 734.
Starns, J. J., \& Ratcliff, R. (2010). The effects of aging on the speedaccuracy compromise: Boundary optimality in the diffusion model. Psychology and Aging, 25(2), 377.

Strickland, L., Elliott, D., Wilson, M. D., Loft, S., Neal, A., \& Heathcote, A. (2019). Prospective memory in the red zone: Cognitive control and capacity sharing in a complex, multi-stimulus task. Journal of Experimental Psychology: Applied.

Strickland, L., Loft, S., Remington, R. W., \& Heathcote, A. (2018). Racing to remember: A theory of decision control in event-based prospective memory. Psychological Review, 125(6), 851.

Touron, D. R., \& Hertzog, C. (2004). Distinguishing age differences in knowledge, strategy use, and confidence during strategic skill acquisition. Psychology and Aging, 19(3), 452.

Uttl, B. (2008). Transparent meta-analysis of prospective memory and aging. PLoS One, 3(2), e1568.

Uttl, B. (2011). Transparent meta-analysis: does aging spare prospective memory with focal vs. non-focal cues?. PloS One, 6(2), e16618.

West, R. L. (1996). An application of prefrontal cortex function theory to cognitive aging. Psychological Bulletin, 120(2), 272.

West, R., Murphy, K. J., Armilio, M. L., Craik, F. I., \& Stuss, D. T. (2002). Lapses of intention and performance variability reveal agerelated increases in fluctuations of executive control. Brain and Cognition, 49(3), 402-419.

Publisher's note Springer Nature remains neutral with regard to jurisdictional claims in published maps and institutional affiliations. 